\title{
Subsurface Sensing of Buried Objects Under a Randomly Rough Surface Using Scattered Electromagnetic Field Data
}

\author{
Reza Firoozabadi, Student Member, IEEE, Eric L. Miller, Senior Member, IEEE, \\ Carey M. Rappaport, Fellow, IEEE, and Ann W. Morgenthaler
}

\begin{abstract}
This paper proposes a new inverse method for microwave-based subsurface sensing of lossy dielectric objects embedded in a dispersive lossy ground with an unknown rough surface. An iterative inversion algorithm is employed to reconstruct the geometry and dielectric properties of the half-space ground as well as that of the buried object. B-splines are used to model the shape of the object as well as the height of the rough surface. In both cases, the control points for the spline function represent the unknowns to be recovered. A single-pole rational transfer function is used to capture the dispersive nature of the background. Here, the coefficients in the numerator and denominator are the unknowns. The approach presented in this paper is based on the state-of-the-art semianalytic mode matching forward model, which is a fast and efficient algorithm to determine the scattered electromagnetic fields. Numerical experiments involving twodimensional geometries and TM incident plane waves demonstrate the accuracy and reliability of this inverse method.
\end{abstract}

Index Terms-B-splines, dispersive media, ground-penetrating radar (GPR), inversion methods, nonlinear optimization, rough surface, subsurface sensing.

\section{INTRODUCTION}

W E ADDRESS the problem of reconstructing the geometry and complex permittivity of a lossy object buried in a dispersive lossy half-space with an unknown rough surface from a noisy collection of scattered electromagnetic field data. Subsurface sensing has been an interesting research area of late with a broad range of applications from environmental monitoring [1]-[3] to nondestructive testing [4]-[6]. A variety of methods have been provided in recent years to solve this category of problems in the frequency or time domain [7]-[16]. Many of these approaches are fully statistical [17]-[19] and are proper for the detection of objects but are not suitable for localization and characterization of them.

When addressing this later problem, two classes of processing techniques are usually considered. Pixel-based inverse methods estimate the unknown physical properties of the medium over a dense tessellation of the region of interest [8], [9], [12]. Where the data are few compared to the number of pixels, as is the case in the severely limited view problems

Manuscript received January 17, 2006; revised May 22, 2006. This work was supported in part by the Center for Subsurface Sensing and Imaging Systems under the Engineering Research Centers Program of the National Science Foundation (Award Number EEC-9986821).

The authors are with the Center for Subsurface Sensing and Imaging Systems, Department of Electrical and Computer Engineering, Northeastern University, Boston, MA 02115 USA (e-mail: rfirooza@ece.neu.edu).

Digital Object Identifier 10.1109/TGRS.2006.883462 considered here, such an approach requires the solution of a large scale and ill-posed inverse problem with all of the attendant issues associated with regularization, e.g., choice of parameter [20]-[22].

Alternatively, when the fundamental problem of interest is the identification of a target, there has been a significant work over the past decade in the use of geometric inverse methods [9], [14]-[16], [23], [24]. The idea here is to replace the large number of pixels with a far smaller set of unknowns, capturing what we really seek from the data: the location, size, shape, orientation, and contrast of the object. The methods we discuss in this paper fall into this class of imaging techniques. The two primary contributions of this paper are: 1) the development of a geometric approach based on a new forward model [25], [26] of low computational complexity, which is particularly well adapted to the problem of imaging in a half-space as well as 2) the joint reconstruction of the geometry and dielectric properties of both the target and the dispersive background.

Inverse methods typically rely on forward solvers based on numerical or analytical models of the wavefield physics. Numerical methods such as finite difference time-domain [27] and the moment method [28], [29] tend to be slow and require excessively large matrix storage, while analytic simulations can often be difficult to implement for problems with more complicated geometries. In this paper, we consider a hybrid model, the semianalytic mode matching method (SAMM) [25], [26], which combines the physical basis of analytic methods with the flexibility and versatility of numerical methods. This model is of low computational complexity compared to other approaches and highly accurate in the region of interest. Moreover, its analytical structure makes it quite well suited for use in an inverse algorithm where sensitivity information (i.e., gradients of the fields with respect to the unknown geometric and contrast parameters) is required.

In our approach, dispersion is parameterized by a singlepole conductivity model [30] valid in at least one decade of frequency, giving us the ground parameters for the whole frequency band we are dealing with, from the data available in just a few frequencies.

These physical models for wave propagation and dispersion are employed in the context of an inverse method for characterizing both the geometry of the object as well as that of the rough interface. When considering the question of shape parameterization, there are options such as Fourier descriptors [31], Lagrange Interpolation [32], and parametrically defined shapes 
[33], [34] based on global representation of the geometry as well as B-splines or snakes [35]-[37] where the unknowns control basically local properties of the curve. In this paper, we have chosen to employ B-splines both to represent the object and the interface.

This choice is made primarily in acknowledgment of the nature of the problem we address in which noisy data are collected in a limited view geometry, and the embedding region is lossy. As such, it is observed in literature that: 1) recovery of fine scale "sharp features" such as corners of objects is for all practical purposes impossible and 2) for all but the smallest objects, the accuracy of the reconstruction is far from uniform in that structure of the object closer to the interface (i.e., the top of the target) is recovered more accurately than that further away (i.e., the bottom) [38]-[40]. As such, the geometric parameterization we seek should be smooth and should provide local control over the shape. B-splines satisfy both of these criteria. We note that the requirements for local control are not as pressing in the case of the rough surface, which is illuminated uniformly by the incident field. Hence, we could have considered other parameterization such as Fourier or Lagrange; however, for ease of implementation, we use B-splines here as well.

The combination of the physical models and geometric parameterizations is, we feel, a contribution to the field of subsurface sensing. More specifically, as shown in Section VI, an extensive set of simulation studies is presented demonstrating the robustness of this method for recovering size, shape, and contrast of objects of varying sizes and geometric characteristics located even under quite rough interfaces. In cases where the object is smoother, the geometric reconstruction is basically exact. For objects with sharp corners, the estimated shape, although less accurate, still provides the basic information of interest in problems such as these.

The remainder of this paper is organized as follows. In Section II, the inverse problem and its formulation are stated. Section III gives a short review of the forward model. Section IV is devoted to parameterization of the boundaries and dielectric properties of the media. Inversion algorithm is fully described in Section V. Section VI details the results of the numerical experiments to verify the computational capability and the efficiency of our inverse method. Conclusions are described in Section VII.

\section{Problem Statement}

The geometry of our problem is given in Fig. 1, demonstrating the lossy dielectric object buried under the rough interface of the air and the lossy dielectric ground. The ground rough surface is illuminated by a multifrequency uniform plane-wave incident at different angles. For the TM case $\left(H_{z}=0\right)$, the incident uniform plane wave, which is to be expressed as a superposition of cylindrical modes, is given by

$$
E_{I z}(x, y)=E_{0} e^{i\left(k_{a x} x-k_{a y} y\right)}
$$

where $E_{0}$ is the electric-field amplitude in the general coordinate system with origin at the midpoint of the nominal flat ground surface, and $k_{a x}$ and $k_{a y}$ are the incident-wave-vector components in the air [41]. For 2-D scattering, an incident TM

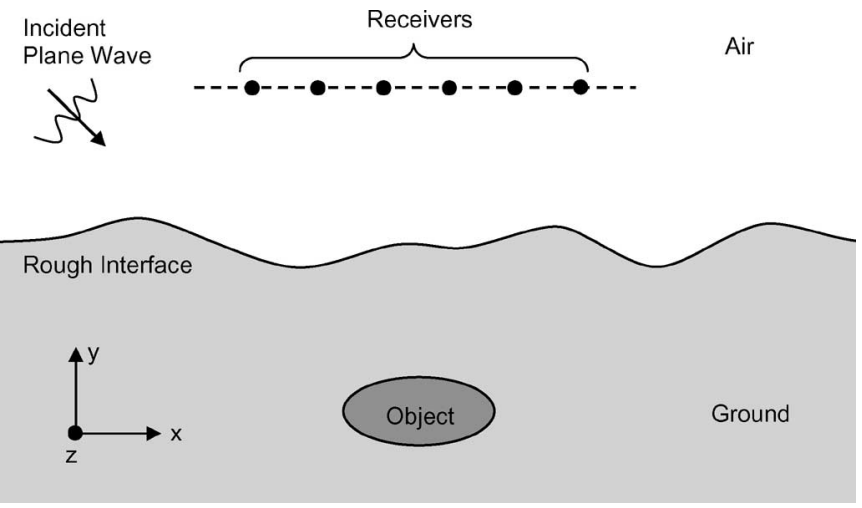

Fig. 1. Problem geometry. Two-dimensional problem where a rough surface separates the air and the ground with the object buried beneath the interface. Receivers are located on a measurement level above the interface. A plane wave is incident on the interface in different angles.

field will give rise only to TM scattering components even in the presence of a rough surface and embedded object.

The electromagnetic field components satisfy the Maxwell equations and radiation condition in the different media, each of which is characterized by their possibly complex dielectric constant $\epsilon$ and permeability $\mu$. The governing homogenous wave equations in dielectric media without free sources are [41]

$$
\begin{aligned}
& \left(\nabla^{2}+k^{2}\right) \mathbf{E}(x, y)=0 \\
& \left(\nabla^{2}+k^{2}\right) \mathbf{H}(x, y)=0
\end{aligned}
$$

where $\nabla^{2}$ is the 2-D cylindrical Laplacian operator, and $\mathbf{E}$ and $\mathbf{H}$ are electric and magnetic field vectors, respectively, with time-harmonic dependence $\exp (-i \omega t)$ and angular frequency $\omega$ for which the dispersion relation $k^{2}=\omega^{2} \mu \epsilon$ must be obeyed.

The fields scattered by the object and ground surface satisfying (2a) and (2b), which are functions of the geometry and media properties, are detected by the receivers located on a measurement level above the air-ground interface. In our inversion algorithm, we attempt to find the unknown parameters by reducing the misfit between the observed data and data predicted by forward model. In other words, our iterative inverse algorithm minimizes a cost function based on the least square formulation as follows:

$$
e(\mathbf{u})=\frac{1}{2}\left\|\mathbf{f}(\mathbf{u})-\mathbf{f}_{\mathbf{0}}\right\|_{2}^{2}=\frac{1}{2} \mathbf{r}(\mathbf{u})^{\mathrm{H}} \mathbf{r}(\mathbf{u})
$$

where $\mathbf{r}=\mathbf{f}(\mathbf{u})-\mathbf{f}_{0}$ is the residual vector, $\mathbf{f}(\mathbf{u})$ is the scattered-field vector calculated by forward solver at the receivers locations for the predicted geometry and contrast, $\mathbf{u}$ is the unknown vector to be described in Section $\mathrm{V}, \mathbf{f}_{0}$ is the noisy observed scattered-field data vector, and $\mathbf{r}^{\mathrm{H}}$ is the transposed complex conjugated vector of $\mathbf{r}$. Mathematically, we seek a $\hat{\mathbf{u}}$ satisfying

$$
\hat{\mathbf{u}}=\arg \min _{\mathbf{u}} e(\mathbf{u}) .
$$

In the following sections, after discussing the forward model and parameterizing the problem, we develop an algorithm to solve this optimization problem in order to optimize the unknowns describing the boundaries and media dielectric properties. For multifrequency and multiangle case, the 
forward problem is solved for all frequencies and incidence angles in each step to obtain the total field vector

$$
\mathbf{f}(\mathbf{u})=\left[\mathbf{f}^{(1)}(\mathbf{u})^{\mathrm{T}}, \mathbf{f}^{(2)}(\mathbf{u})^{\mathrm{T}}, \ldots, \mathbf{f}^{\left(N_{c}\right)}(\mathbf{u})^{\mathrm{T}}\right]^{\mathrm{T}}
$$

where $N_{c}$ is the total number of frequencies and incidence angles.

Our subsurface-sensing inverse method uses the SAMM forward model to relate the scattered fields to unknown structure and media dielectric properties, which are parameterized in compact form. Unknown parameters are optimized to fit the calculated scattered fields to observed data.

\section{FORWARD MODEL}

In this section, we will provide sufficient details concerning our forward model to understand how it can be used in the associated inversion scheme. The full explanation of SAMM can be found in [26].

Our forward model aims to solve the scattering problem in the presence of the incident plane waves [42]. For a circular object in infinite soil background, the scattered waves can be described by conventional analytic Mie scattering formulas [43]. However, for noncircular buried objects under a rough ground plane, there are additional non-Mie and rescattering modes (r-modes) describing the deviation from the Mie solution. The essence of the SAMM method is to use Green's function-type expansions of the non-Mie fields in each region (air, earth, object) for a number of so-called coordinate scattering centers (CSCs). As illustrated in Fig. 2, we employ two CSCs in our problem: one located at the center of the object and the second at its image point above the interface. As explained in [25], associated with each CSC are three superpositions of modes called mode families: one each for air, ground, and the buried object. For our problem, the SAMM solver would employ six mode expansions (three families for each of two CSCs). It turns out, however, that the image CSC in our problem is only required to represent the reflection of rescattered modes from the surface to the medium beneath so that no air modes are needed [26]. Hence, for our problem, five mode families are employed. The mode functions are built such that Maxwell equations are satisfied within each medium. Due to the finite number of modes in each family, the coefficients of the expansions are chosen to satisfy the boundary conditions in a least square sense.

With judicious use of the Bessel and Hankel relations, we can find the Cartesian field components, each of which must satisfy the scalar wave equation for each CSC, such that for TM modes [26]

$$
\begin{aligned}
& H_{x}\left(x^{\prime}, y^{\prime}\right)=\lim _{N_{T} \rightarrow \infty} \sum_{n=-N_{T}}^{N_{T}} \frac{\left(c_{n-1}+c_{n+1}\right)}{2 \eta} G_{n}\left(x^{\prime}, y^{\prime}\right) \\
& H_{y}\left(x^{\prime}, y^{\prime}\right)=\lim _{N_{T} \rightarrow \infty} \sum_{n=-N_{T}}^{N_{T}} \frac{\left(c_{n-1}-c_{n+1}\right)}{2 i \eta} G_{n}\left(x^{\prime}, y^{\prime}\right) \\
& E_{z}\left(x^{\prime}, y^{\prime}\right)=\lim _{N_{T} \rightarrow \infty} \sum_{n=-N_{T}}^{N_{T}} c_{n} G_{n}\left(x^{\prime}, y^{\prime}\right)
\end{aligned}
$$

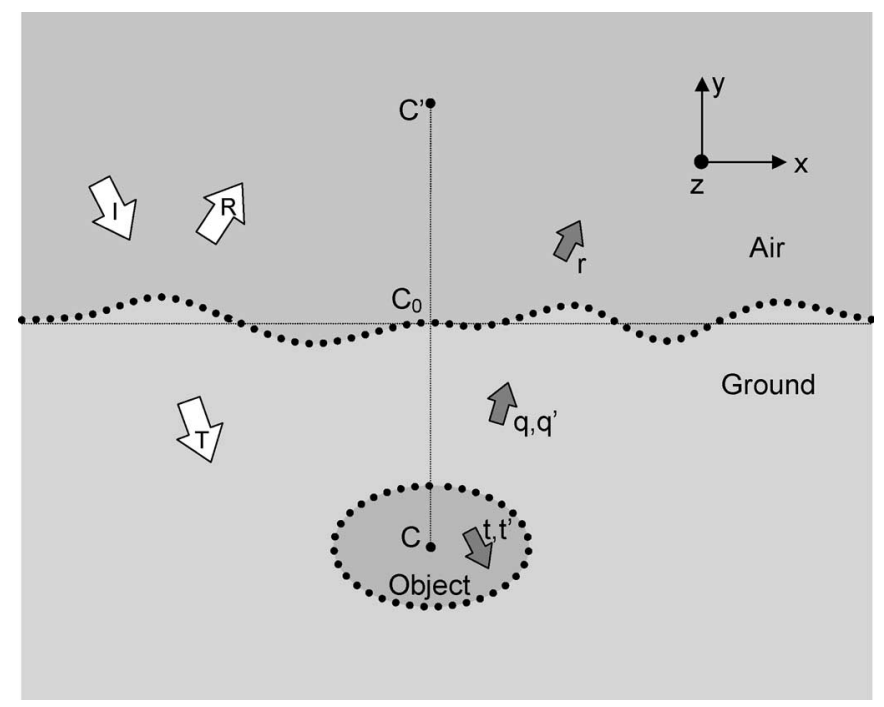

Fig. 2. Circular object under a random rough ground surface. $C$ and $C^{\prime}$ represent CSCs of the object and its image about the nominal flat ground surface. Incident, reflected, and transmitted plane waves are given by $I, R$, and $T$, respectively. Mode families are $t$ and $t^{\prime}$ including r-modes and Mie modes within the object originating at the object center and its image, respectively, $q$ and $q^{\prime}$ including r-modes and Mie modes within the ground originating at the object center and its image, respectively, and $r$ including r-modes originating at the object center. Modes in the regions including their scattering centers are defined by the Bessel functions of the first kind $\left(J_{n}\right)$; otherwise the Hankel functions of the first kind $\left(H_{n}^{(1)}\right)$ are used.

with $c_{n}$ as the cylindrical mode coefficient, $\eta$ as the wave impedance, and

$$
G_{n}\left(x^{\prime}, y^{\prime}\right)=F_{n}(k \rho) e^{i n \phi}
$$

where $x^{\prime}$ and $y^{\prime}$ indicate the coordinates of the point with $x^{\prime}=\rho \cos \phi$ and $y^{\prime}=\rho \sin \phi$ in local coordinate system and scattering center at object center or its image. $F_{n}(k \rho)$ is Bessel or Hankel function of integer order $n$ such that

$$
F_{n}(k \rho)= \begin{cases}J_{n}(k \rho), & \text { CSC inside the region } \\ H_{n}^{(1)}(k \rho), & \text { CSC outside the region. }\end{cases}
$$

In the SAMM algorithm, we first find mode coefficients satisfying the boundary conditions on the ground surface and object boundary. These mode coefficients are then used to obtain scattered fields at specified receiver points.

Superposing the modes arising from each CSC gives rise to the scattered fields at any point of the space in our region of interest. Applying the superposition of the modes (6a)-(6c) above and below each point on a boundary (as indicated by dots in Fig. 2) and enforcing boundary conditions on all field components at those points lead to an overdetermined matrix equation of the form $\mathbf{L c}=\mathbf{b}$, where $\mathbf{c}$ is the mode coefficient vector, $\mathbf{b}=\left[\mathbf{b}_{1}^{\mathrm{T}}, \mathbf{b}_{2}^{\mathrm{T}}\right]^{\mathrm{T}}$ is the field difference vector on the boundaries caused by refraction of the incident plane wave [26], and $\mathbf{L}$ is the matrix linking the unknown mode coefficients and the scattered cylindrical modes. Since $\mathbf{L}$ is nonsquare, matrix inversion is performed by singular value 
decomposition (SVD) minimizing $\|\mathbf{b}-\mathbf{L} \mathbf{c}\|_{2}^{2}$, which leads to the result

$$
\mathbf{c}=\mathbf{L}^{+} \mathbf{b}
$$

where $\mathbf{L}^{+}$is the pseudoinverse of $\mathbf{L}$.

The summations in (6a)-(6c) are terminated at a finite value resulting in a finite number of mode coefficients. In detail, the vector $\mathbf{c}=\left[\mathbf{c}^{1}, \mathbf{c}^{2}, \ldots, \mathbf{c}^{5}\right]^{\mathrm{T}}$ is the unknown coefficient vector including all mode-family vectors

$$
\mathbf{c}^{p}=\left[c_{-N_{T}}^{p}, c_{-N_{T}+1}^{p}, \ldots, c_{N_{T}}^{p}\right]
$$

where $p=1, \ldots, 5$, and the truncated number of modes in each mode family is $2 N_{T}+1$.

The matrix $\mathbf{L}$ is defined as the block matrix

$$
\mathbf{L}=\left[\begin{array}{llll}
\mathbf{L}_{1}^{(1)} & \mathbf{L}_{2}^{(1)} & \cdots & \mathbf{L}_{5}^{(1)} \\
\mathbf{L}_{1}^{(2)} & \mathbf{L}_{2}^{(2)} & \cdots & \mathbf{L}_{5}^{(2)}
\end{array}\right]
$$

where superscripts (1) and (2) indicate the ground and object boundaries, respectively.

The matrix $\mathbf{L}_{m}^{(i)}(i=1,2$ and $m=1,2, \ldots, 5)$ is nonzero for some mode families and is equal to

$$
\mathbf{L}_{m}^{(i)}=\left[\begin{array}{lll}
\mathbf{L}_{x, m}^{(i)}{ }^{\mathrm{T}} & \mathbf{L}_{y, m}^{(i)}{ }^{\mathrm{T}} & \mathbf{L}_{z, m}^{(i)}{ }^{\mathrm{T}}
\end{array}\right]^{\mathrm{T}} .
$$

The matrices $\mathbf{L}_{x, m}^{(i)}, \mathbf{L}_{y, m}^{(i)}$, and $\mathbf{L}_{z, m}^{(i)}$ are of dimension $N_{s}^{(i)} \times$ $\left(2 N_{T}+1\right)$, where $N_{s}^{(i)}$ is the number of points on $i$ th surface, and $N_{T}$ is the truncated number of modes in each family.

The elements of these matrices at each point $\left(x^{\prime}, y^{\prime}\right)$ on the $i$ th surface for $n$th mode in the $m$ th mode-family coordinate system are given by

$$
\begin{aligned}
& L_{x}\left(x^{\prime}, y^{\prime}\right)=\left(G_{n-1}\left(x^{\prime}, y^{\prime}\right)+G_{n+1}\left(x^{\prime}, y^{\prime}\right)\right) / 2 \eta \\
& L_{y}\left(x^{\prime}, y^{\prime}\right)=\left(G_{n-1}\left(x^{\prime}, y^{\prime}\right)-G_{n+1}\left(x^{\prime}, y^{\prime}\right)\right) / 2 i \eta \\
& L_{z}\left(x^{\prime}, y^{\prime}\right)=G_{n}\left(x^{\prime}, y^{\prime}\right) .
\end{aligned}
$$

The scattered fields at the receivers above the air-ground interface are also obtained using (6a)-(6c) as

$$
\mathbf{f}=\mathbf{M c}
$$

where the matrix $\mathbf{M}$ links the mode coefficient vector $\mathbf{c}$ to the scattered-field vector $\mathbf{f}=\left[\mathbf{f}_{x}^{\mathrm{T}}, \mathbf{f}_{y}^{\mathrm{T}}, \mathbf{f}_{z}^{\mathrm{T}}\right]^{\mathrm{T}}$ at the receiver locations. In TM case, the elements of the vectors $\mathbf{f}_{x}, \mathbf{f}_{y}$, and $\mathbf{f}_{z}$ are the field component $H_{x}\left(x^{\prime}, y^{\prime}\right), H_{y}\left(x^{\prime}, y^{\prime}\right)$ and $E_{z}\left(x^{\prime}, y^{\prime}\right)$, respectively, where $\left(x^{\prime}, y^{\prime}\right)$ indicates the location of the receivers in local coordinate system.

Combining (9) and (14), the scattered-field vector at the receivers in general form is given by

$$
\mathbf{f}=\mathbf{M L}^{+} \mathbf{b} .
$$

In our problem, we have only one nonzero family mode at the receiver locations in the air, which is originating at the object center. The matrix $\mathbf{M}$ is

$$
\mathbf{M}=\left[\begin{array}{lll}
\mathbf{M}_{x}^{\mathrm{T}} & \mathbf{M}_{y}^{\mathrm{T}} & \mathbf{M}_{z}^{\mathrm{T}}
\end{array}\right]^{\mathrm{T}} .
$$

The matrices $\mathbf{M}_{x}, \mathbf{M}_{y}$, and $\mathbf{M}_{z}$ are of dimension $N_{\mathrm{r}} \times$ $\left(2 N_{T}+1\right)$, where $N_{\mathrm{r}}$ is the number of receiver points. The elements of these matrices are identical to (13a)-(13c) at each receiver point $\left(x^{\prime}, y^{\prime}\right)$ in each mode-family coordinate system.

The significant point is that all right-hand-side variables of (15) depend on the location of boundary points and, hence, on the parameters defining the shape of the object and surface. As we discuss next, our inverse method makes use of an analytically tractable parameterization of geometry that can be exploited in an inversion routine.

\section{Parameterization of the Problem}

\section{A. Geometry Parameterization}

To define the surfaces in low-dimensional parametric form, we model them by B-splines, which are piecewise polynomial functions providing local approximation to curves using a small finite number of parameters called control points. A curve is defined in B-spline parametric form with basic functions associated with $N_{u}$ control points $\mathbf{p}_{i}$ as [36]

$$
\mathbf{P}(t)=\sum_{i=1}^{N_{u}} \mathbf{p}_{i} N_{i, k}(t) \quad \text { for } \quad t_{k} \leq t<t_{N_{u}+1}
$$

with the basis functions $N_{i, k}(t)$ defined by the recurrence relations

$$
\begin{aligned}
& N_{i, 1}(t)= \begin{cases}1, & \text { if } t_{i} \leq t<t_{i+1} \\
0, & \text { otherwise }\end{cases} \\
& N_{i, k}(t)=\frac{t-t_{i}}{t_{i+k-1}-t_{i}} N_{i, k-1}(t)+\frac{t_{i+k}-t}{t_{i+k}-t_{i+1}} N_{i+1, k-1}(t)
\end{aligned}
$$

where $T=\left\{t_{1}, t_{2}, \ldots, t_{N_{u}+k}\right\}$ is called the knot vector with $t_{1}<t_{2}<\cdots<t_{N_{u}+k}$.

Each $N_{i, k}(t)$ is a polynomial of order $k$ (degree $k-1$ ), which is $k-2$ times differentiable and continuous $\left(C^{k-2}\right)$ on each interval. The basis functions are entirely determined by the relative distance between the knots, so their shapes are unaffected by scaling or shifting them together. In the case with uniform knot vector for which $t_{i+1}-t_{i}\left(i=1,2, \ldots, N_{u}\right)$ is constant, all basis functions are just shifted copy of each other. For simplicity, we define the uniform knot vector by $T_{i}=i$.

The order $k$ of the B-splines is the difference between the number of knots and control points. Increasing the B-spline order increases the smoothness of the curve. The B-spline curve $\mathbf{P}(t)$ is defined to be valid only in the range of $t_{k} \leq t<$ $t_{N_{u}+1}$, where the basis functions provide the partition of unity property: $\sum_{i=1}^{N_{u}} N_{i, k}(t)=1$. The ends of a curve can be joined to make a closed loop. In order to keep the $C^{k-2}$ continuity of the closed curve, the first $k-1$ control points must repeat at the end [37].

The shape of a B-spline curve can be changed by modifying a number of parameters such as the position, number, or multiplicity of the control points, the number, relative spacing, or multiplicity of the knots, or the order of B-splines. In this paper, we change the shape of the curves by adjusting their controlpoint locations and keep the other parameters fixed. 


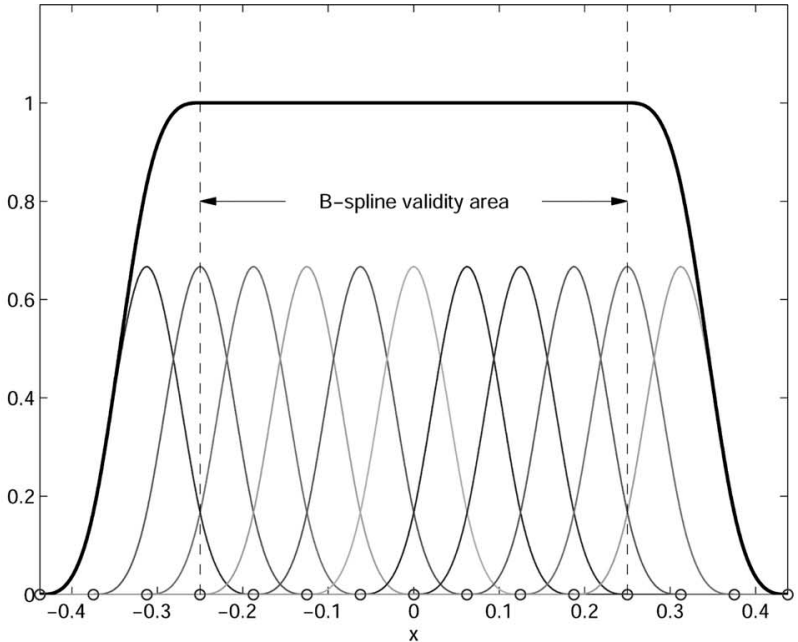

(a)

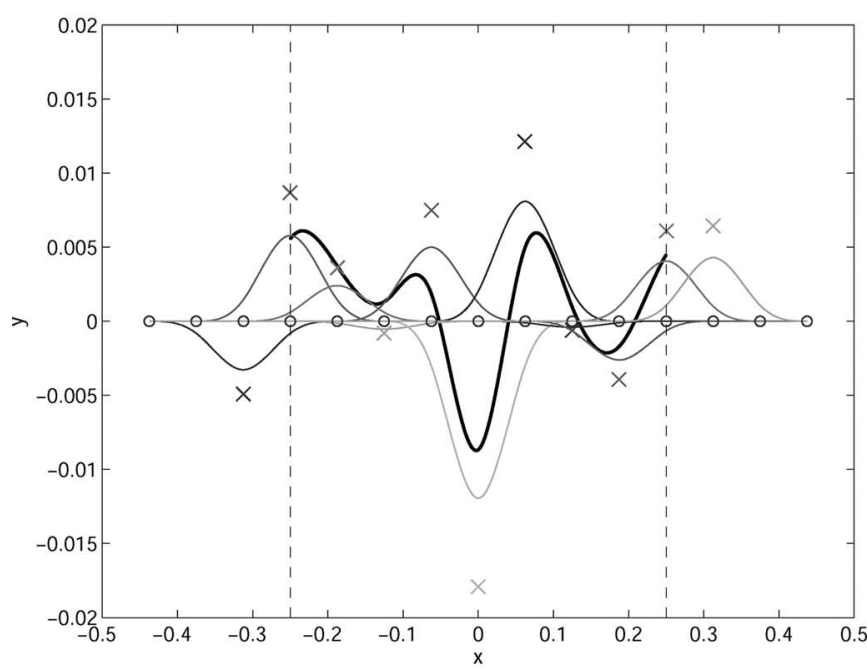

(b)

Fig. 3. Creating ground surface by a B-spline curve. (a) Basis functions (thin lines) and their summation (bold line) versus $x$; knots are indicated by "०." (b) Basis functions weighted by control points (thin lines) and final curve (bold line) versus $x$. Control points are indicated by " $\times$."

As an example, to construct a ground surface using cubic B-spline formulation for the variable $y$ (the height) at given $x$ positions with, e.g., 11 control points, we define $\mathbf{p}_{i}=\left[p_{y, i}\right]$, $i=1,2, \ldots, 11$. For a cubic B-spline $(k=4)$, the knot vector consists of 15 elements distributed uniformly along $x$ axis. Fig. 3(a) shows the basis functions and their summation versus $x$ with the region of validity of the curve. The final curve is the superposition of the basis functions weighted by their associated control points illustrated in Fig. 3(b).

The object is modeled by a closed cubic B-spline. To define a closed cubic B-spline with say six control points, the first three control points repeat at the end such that $\mathbf{p}_{i}=\left[p_{x, i}, p_{y, i}\right]^{\mathrm{T}}$, and $i=1,2, \ldots, 9$, where $\mathbf{p}_{j}=\mathbf{p}_{j+6}, j=1,2,3$. The knot vector consists of 13 elements distributed uniformly versus polar angle. Fig. 4(a) shows the basis functions and their summation with the region of validity of the curve. The basis functions weighted by the $x$ and $y$ components of their associated control points and their superposition versus knots are shown in Fig. 4(b) and (c), respectively. The final closed curve in $x-y$ plane is illustrated in Fig. 4(d).

\section{B. Dielectric-Property Parameterization}

The dispersive soil has frequency-dependent complex permittivity, and we need a parametric model that models its behavior within the desired frequency range. A model that displays good agreement with the dispersive soil data over more than a decade of frequency was developed in [30] and is referred to as a single-pole conductivity model. We employ this model in this paper. The single-pole conductivity model defines the complex conductivity as

$$
\sigma_{g}=\frac{b_{0}+b_{1} z^{-1}+b_{2} z^{-2}}{1+a_{1} z^{-1}}
$$

where $b_{0}, b_{1}, b_{2}$, and $a_{1}$ are the model parameters, and $z=$ $e^{-i \omega \Delta T}$ with the constant $\Delta T$.

The complex permittivity is then defined by

$$
\epsilon_{g}(\omega)=\epsilon_{a v} \epsilon_{0}+i \frac{\sigma_{g}}{\omega}=\epsilon_{a v} \epsilon_{0}+\frac{i\left(b_{0}+b_{1} z^{-1}+b_{2} z^{-2}\right)}{\omega\left(1+a_{1} z^{-1}\right)}
$$

where $\epsilon_{a v}$ is a frequency-independent parameter.

\section{INVERSION ALGORITHM}

There are a variety of gradient descent methods to solve the problems of the form of nonlinear least square minimization. Among all these methods, we choose the Levenberg-Marquardt algorithm [44], [45]. This method can be considered as a trustregion modification of the Gauss-Newton algorithm. It uses a search direction that is between the Gauss-Newton direction and the steepest descent direction. In many cases, it is more effective than some other numerical methods such as steepest descent and conjugate gradients [46].

The unknown vector is

$$
\mathbf{u}=\left[\mathbf{u}_{1}^{\mathrm{T}}, \mathbf{u}_{2}^{\mathrm{T}}, \mathbf{u}_{g}^{\mathrm{T}}, \mathbf{u}_{t}^{\mathrm{T}}\right]^{\mathrm{T}}
$$

where the subscripts $1,2, g$, and $t$ refer to the unknowns pertaining to the ground surface, object surface, ground complex permittivity, and object complex permittivity, respectively. Therefore

$$
\begin{aligned}
\mathbf{u}_{1} & =\left[p_{y, 1}^{(1)}, p_{y, 2}^{(1)}, \ldots, p_{y, N_{u 1}}^{(1)}\right]^{\mathrm{T}} \\
\mathbf{u}_{2} & =\left[p_{x, 1}^{(2)}, p_{x, 2}^{(2)}, \ldots, p_{x, N_{u 2}}^{(2)}, p_{y, 1}^{(2)}, p_{y, 2}^{(2)}, \ldots, p_{y, N_{u 2}}^{(2)}\right]^{\mathrm{T}} \\
\mathbf{u}_{g} & =\left[\epsilon_{a v}, b_{0}, b_{1}, b_{2}, a_{1}\right]^{\mathrm{T}} \\
\mathbf{u}_{t} & =\left[\epsilon_{t r}, \epsilon_{t i}\right]^{\mathrm{T}}
\end{aligned}
$$

The Levenberg-Marquardt algorithm uses the following updating formula to find the updated unknown vector in $n$th step starting from an initial guess $\mathbf{u}^{(0)}$ [47]:

$$
\begin{aligned}
\mathbf{u}^{(n+1)} & =\mathbf{u}^{(n)}-\mathbf{d}^{(n)} \\
\mathbf{d}^{(n)} & =\left(\nabla^{2} e\left(\mathbf{u}^{(n)}\right)+\lambda^{(n)} \mathbf{I}_{N_{u}}\right)^{-1} \nabla e\left(\mathbf{u}^{(n)}\right)
\end{aligned}
$$

where $\nabla e$ and $\nabla^{2} e$ are gradient and Hessian of the cost function, respectively, the matrix $\mathbf{I}_{N_{u}}$ is the identity matrix, $N_{u}$ is the number of unknowns, and $\lambda$ is the Levenberg-Marquardt 


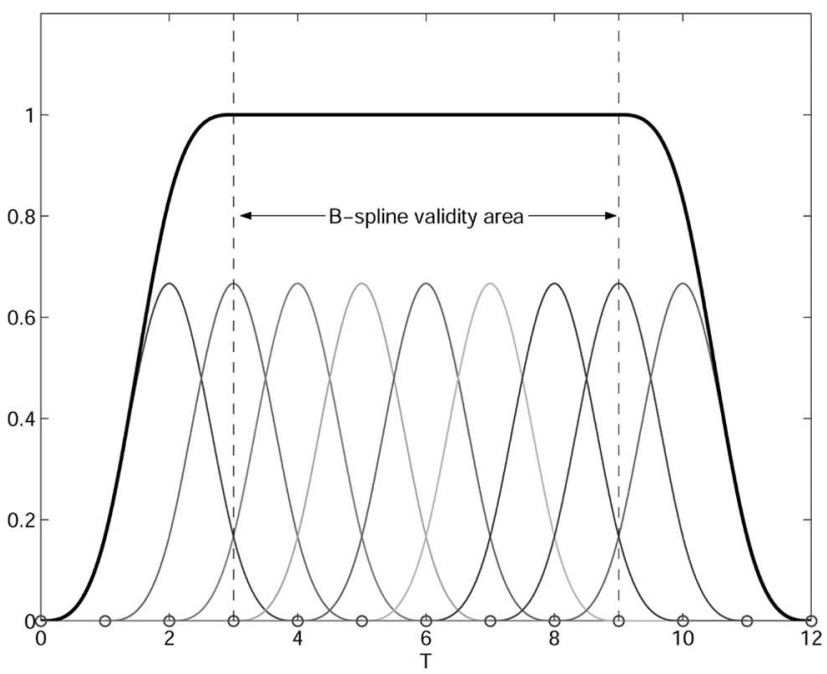

(a)

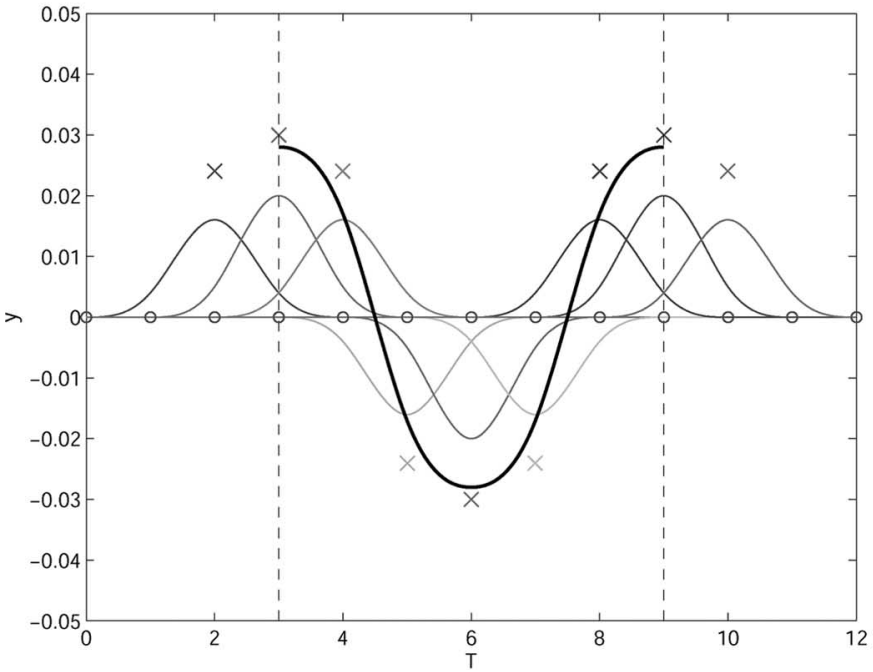

(c)

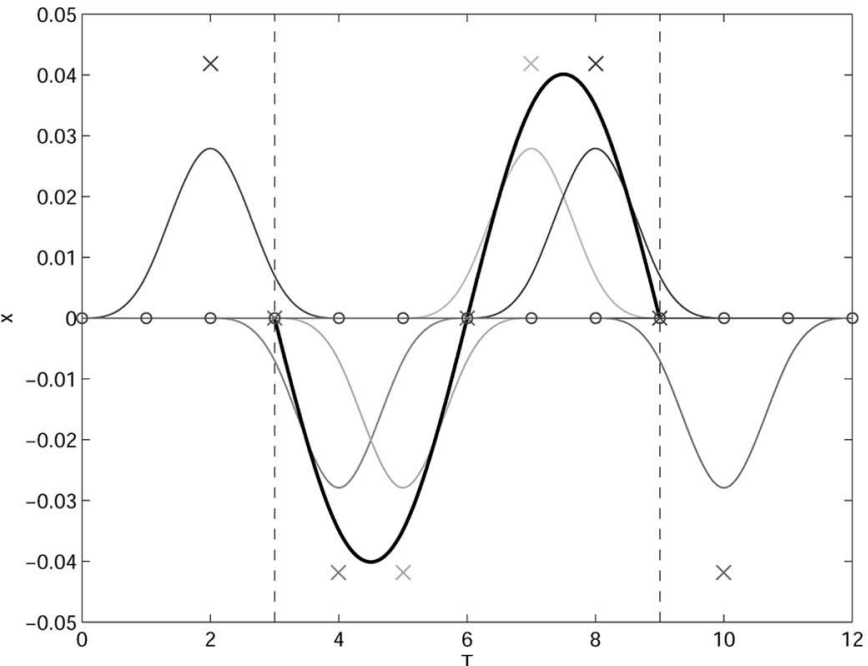

(b)

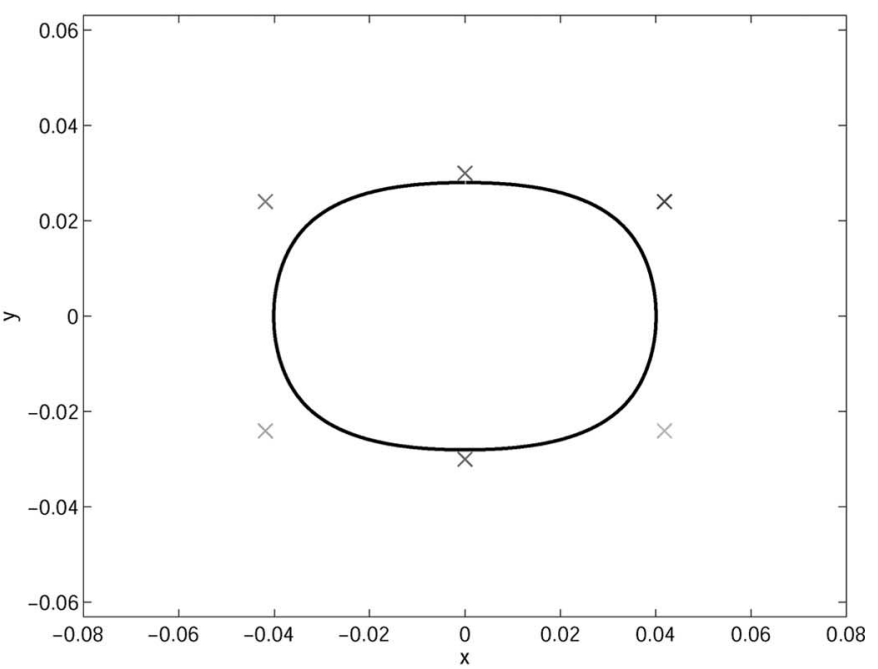

(d)

Fig. 4. Creating object boundary by a closed B-spline curve. (a) Basis functions (thin lines) and their summation (bold line) versus knots. (b) Basis functions weighted by $x$ component of control points (thin lines) and $x$-variation of final curve versus knots (bold line). (c) Basis functions weighted by $y$ component of control points (thin lines) and $y$-variation of final versus knots (bold line). (d) Final closed curve. In all figures, the knots are indicated by "o" and the control points by " $\times$."

parameter being updated in each iteration. Some complexvalued matrix calculations lead to

$$
\begin{aligned}
\nabla e & =\operatorname{Re}\left(\mathbf{J}^{\mathrm{H}} \mathbf{r}\right) \\
\nabla^{2} e & =\operatorname{Re}\left(\mathbf{J}^{\mathrm{H}} \mathbf{J}+\sum_{j=1}^{N_{u}} \nabla^{2} r_{j}^{*} r_{j}\right)
\end{aligned}
$$

where

$$
\mathbf{J}=\frac{\partial \mathbf{r}(\mathbf{u})}{\partial \mathbf{u}}=\frac{\partial \mathbf{f}(\mathbf{u})}{\partial \mathbf{u}}
$$

is the Jacobian matrix of the scattered-field vector. The second term in (26) can be ignored if $\nabla^{2} r_{j}$ or the residuals are small; in this case, we can get the Hessian just by computing the Jacobian matrix such that

$$
\nabla^{2} e \approx \operatorname{Re}\left(\mathbf{J}^{\mathrm{H}} \mathbf{J}\right)
$$

From (14), the derivative of the scattered-field vector is found using matrix differentiation properties as

$$
\frac{\partial \mathbf{f}}{\partial \mathbf{u}}=\frac{\partial(\mathbf{M} \mathbf{c})}{\partial \mathbf{u}}=\mathbf{M} \frac{\partial \mathbf{C}}{\partial \mathbf{u}}+\frac{\partial \mathbf{M}}{\partial \mathbf{u}} \mathbf{C}
$$

where $\mathbf{C}=\mathbf{I}_{N_{u}} \otimes \mathbf{c}$, and $\otimes$ is the Kronecker product operation. On the other hand, from (9)

$$
\frac{\partial \mathbf{b}}{\partial \mathbf{u}}=\frac{\partial(\mathbf{L} \mathbf{c})}{\partial \mathbf{u}}=\frac{\partial \mathbf{L}}{\partial \mathbf{u}} \mathbf{C}+\mathbf{L} \frac{\partial \mathbf{c}}{\partial \mathbf{u}}
$$

or

$$
\frac{\partial \mathbf{c}}{\partial \mathbf{u}}=\mathbf{L}^{+}\left(\frac{\partial \mathbf{b}}{\partial \mathbf{u}}-\frac{\partial \mathbf{L}}{\partial \mathbf{u}} \mathbf{C}\right)
$$

The Jacobian matrix is then obtained as

$$
\mathbf{J}=\mathbf{M L}^{+}\left(\frac{\partial \mathbf{b}}{\partial \mathbf{u}}-\frac{\partial \mathbf{L}}{\partial \mathbf{u}} \mathbf{C}\right)+\frac{\partial \mathbf{M}}{\partial \mathbf{u}} \mathbf{C}
$$




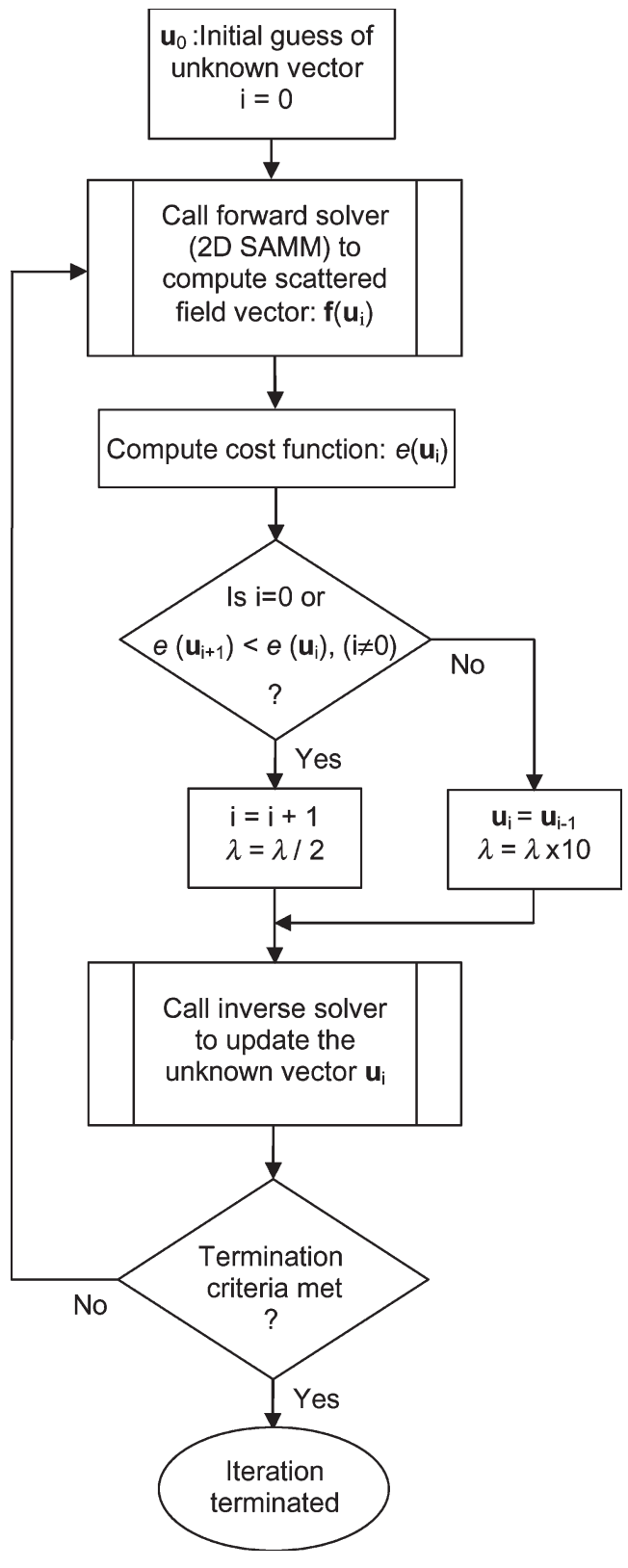

Fig. 5. Inverse-method block diagram.

From this equation, we see that the Jacobian is entirely determined by calculating the elements of the matrices $\partial \mathbf{b} / \partial \mathbf{u}$, $\partial \mathbf{L} / \partial \mathbf{u}$, and $\partial \mathbf{M} / \partial \mathbf{u}$, which is done in the Appendix.

In a problem with a total of $N_{c}$ different frequencies and incidence angles, the total residue vector and Jacobian matrix are defined as

$$
\begin{aligned}
& \mathbf{r}=\left[\mathbf{r}^{(1)^{\mathrm{T}}}, \mathbf{r}^{(2)^{\mathrm{T}}}, \ldots, \mathbf{r}^{\left(N_{c}\right)^{\mathrm{T}}}\right]^{\mathrm{T}} \\
& \mathbf{J}=\left[\mathbf{J}^{(1)^{\mathrm{T}}}, \mathbf{J}^{(2)^{\mathrm{T}}}, \ldots, \mathbf{J}^{\left(N_{c}\right)^{\mathrm{T}}}\right]^{\mathrm{T}}
\end{aligned}
$$

and are used to find the gradient and Hessian utilized in (25) and (28).

A block diagram of the inverse method is demonstrated in Fig. 5. An important issue in the algorithm is updating $\lambda$ appropriately in each step. For small Levenberg-Marquardt

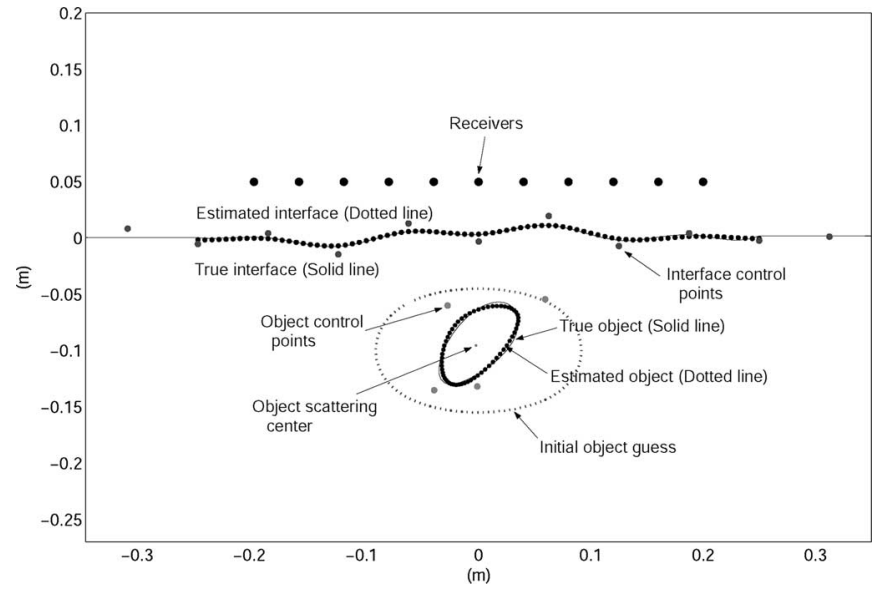

Fig. 6. Problem configuration, initial guess, and reconstructed boundaries with SNR $=30 \mathrm{~dB}$.

parameter values, the scheme approximates the Gauss-Newton method, which is fast but sometimes converges poorly. For large values of $\lambda$, the Levenberg-Marquardt technique resembles steepest descent, which provides slow but certain convergence. Hence, the idea for choosing $\lambda$ is to begin with a sufficiently large value. In each iteration, if the cost function would be increased as a result of an update, which uses the previous value of $\lambda$, the parameter is increased by a factor of ten, and a new update is computed. Otherwise, if the error has decreased as a result of the update, then $\lambda$ is decreased for the next iteration by a factor of two.

\section{NUMERICAL RESUlTS}

In this section, we verify our inverse method by two numerical examples. In the first example, the boundaries and dielectric properties are reconstructed where the source is a normal multifrequency incident plane-wave, and noisy data are collected for a range of noise levels. In the second example, a number of examples have been included, which better highlight both the capabilities as well as the limitations of our method.

In both examples, the reference scattered-field data at the receiver locations for each frequency are simulated via a 2-D finite-difference frequency-domain (FDFD) Matlab code, which calculates the electric and magnetic fields on a discretized spatial grid including the location of the receivers [48].

\section{A. Basic Example}

The geometry of this example is depicted in Fig. 6. The irregular almondlike object is buried shallowly in the ground with burial depth of $10 \mathrm{~cm}$ from the nominal flat interface to the center of the object. The dispersive lossy ground is Bosnian soil with density of $1.26 \mathrm{~g} / \mathrm{cc}$ and moisture of $25 \%$ where its single-pole conductivity-model parameters are shown in Table I [49]. The object is assumed to be lossy and nondispersive with relative complex permittivity of $\epsilon_{t}=2.9(1+i 0.001)$. The rough surface is randomly generated by a Gaussian distribution around the nominal interface. The incident wave is a normally incident multifrequency uniform TM plane wave with zero phase at the origin. The scattered-field data $\left(H_{x}, H_{y}, E_{z}\right)$ are 
TABLE I

Single-Pole Conductivity-Model Parameter VAlues For BOSNIAN SOIL $(d=1.26 \mathrm{~g} / \mathrm{cc}, m=25 \%)$ AND $\Delta T=20 \mathrm{ps}$

\begin{tabular}{|c|c|}
\hline parameter & value \\
\hline$\epsilon_{a v}$ & 5.038 \\
$a_{1}$ & -0.925 \\
$b_{0}$ & 1.76106 \\
$b_{1}$ & -3.32102 \\
$b_{2}$ & 1.56193 \\
\hline
\end{tabular}
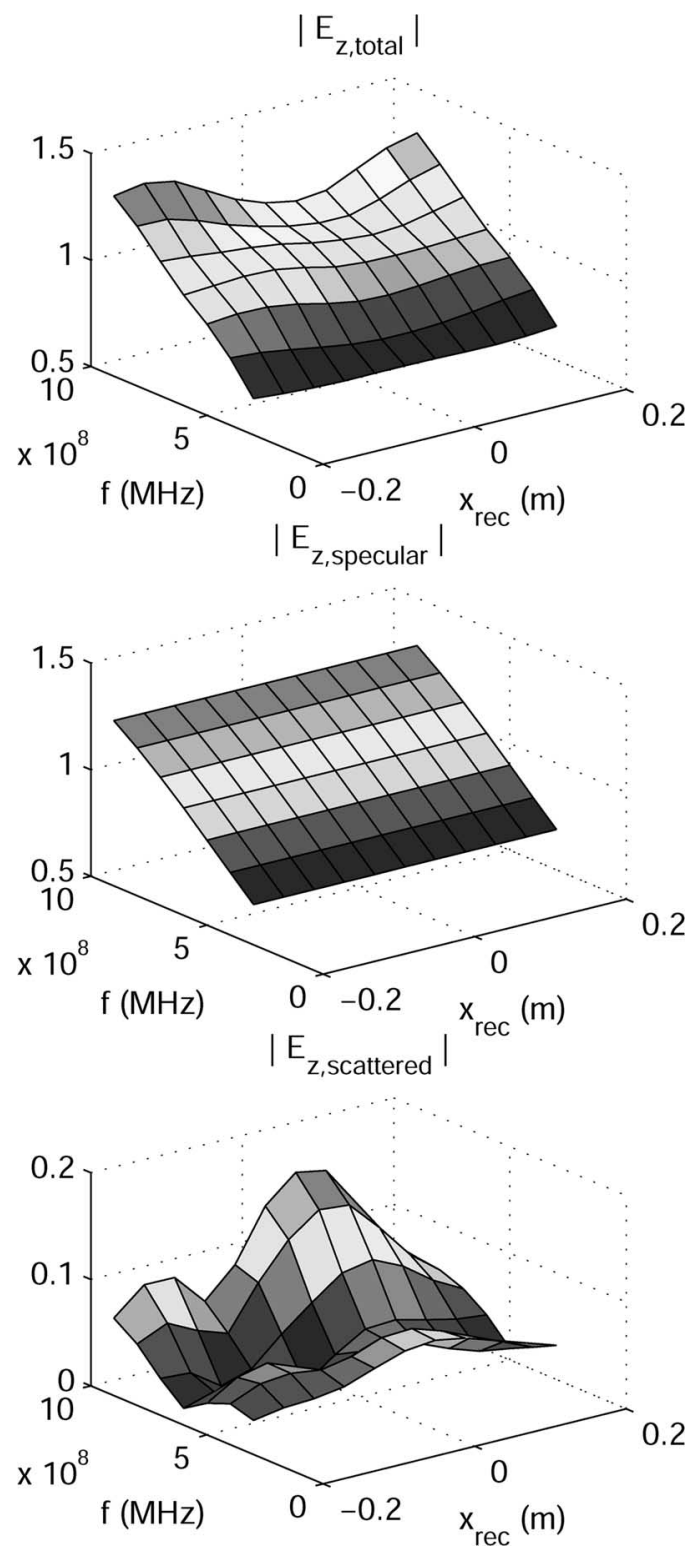

Fig. 7. Magnitude of observed total, specular, and scattered electric fields versus frequency at the receiver locations.

collected at 11 receivers located $5 \mathrm{~cm}$ above the nominal interface and $4 \mathrm{~cm}$ apart from each other. Data available at these points are corrupted with an additive complex Gaussian white noise, which simulates the measurement errors and other variations from actual problem parameters.

We use seven frequencies ranging from $300-900 \mathrm{MHz}$ with $100-\mathrm{MHz}$ frequency resolution. The magnitude of the observed total, specular, and scattered electric fields is depicted in Fig. 7

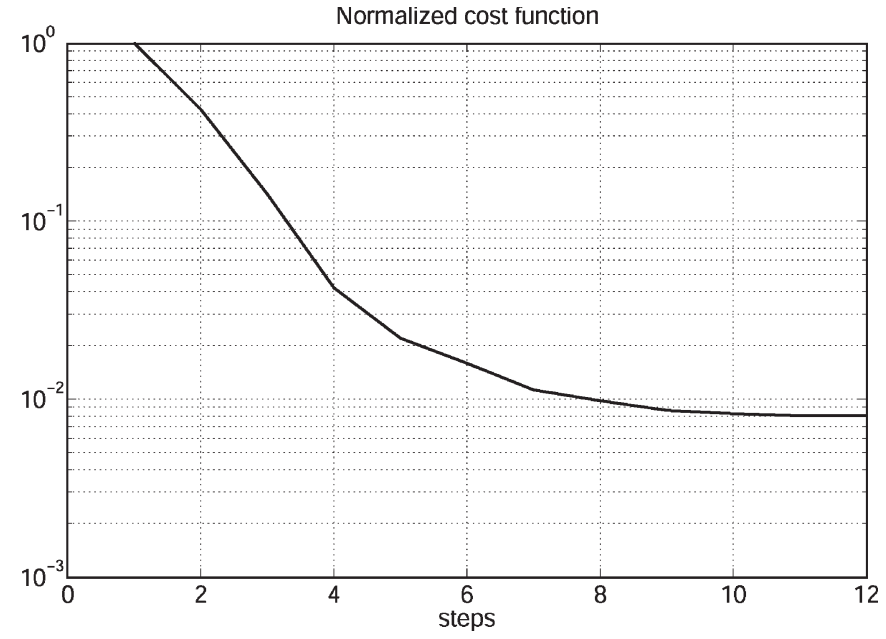

Fig. 8. Normalized cost function versus converging iteration steps for iterative optimization with $\mathrm{SNR}=30 \mathrm{~dB}$.

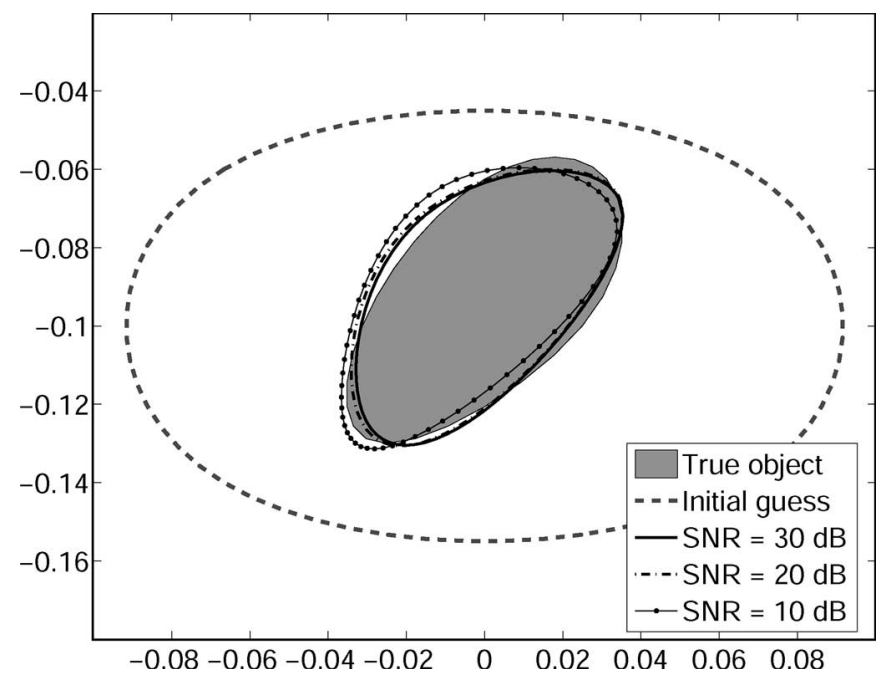

Fig. 9. True object, initial guess, and reconstructed object for three different SNR ratios.

versus the frequency and location of the receivers. The real and imaginary parts of the scattered fields at each frequency are calculated by subtracting the relevant specular field parts from relevant total field parts at receivers.

We make use of nine modes for each mode family in our SAMM forward model. The object maximum dimension is about $1 / 3$ of the wavelength at the highest frequency. The interface and object boundaries are discretized to 81 and 60 points defined by 11 and 4 control points, respectively, where interface control points are one dimensional, and object control points are 2-D. The object and ground complex permittivities are defined by two and five parameters, respectively, according to their parameterization in Section IV-B. Therefore, we have a vector including 26 unknowns to be optimized simultaneously.

The initial guess for air-ground interface is simply a flat surface while the object is initiated from a large closed curve residing in the region of interest, as illustrated in Figs. 6 and 9 . The permittivity initial guess resides within $20 \%$ of the actual value for the object and 50\% of the actual value for the soil. The Levenberg-Marquardt algorithm converges in 


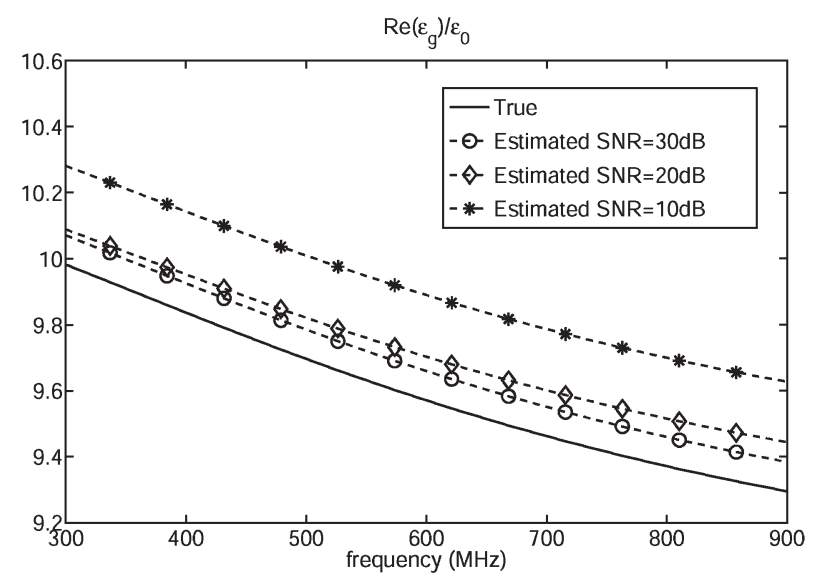

(a)

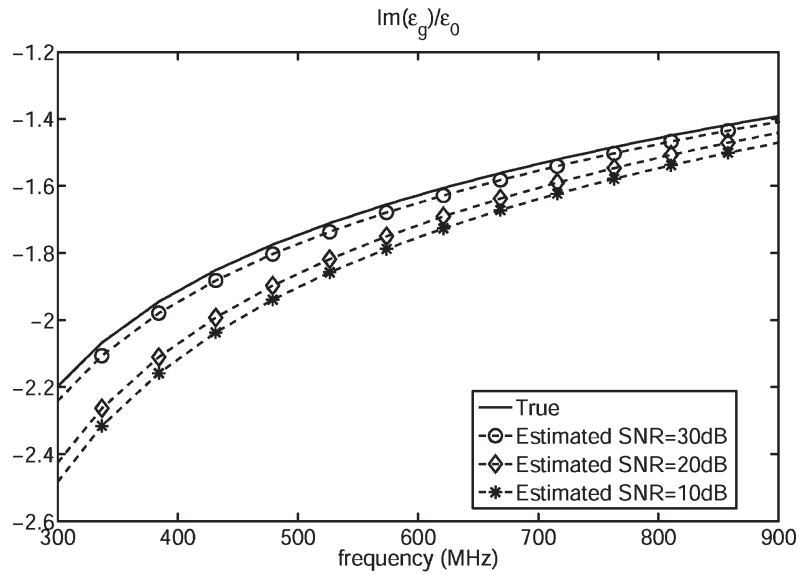

(b)

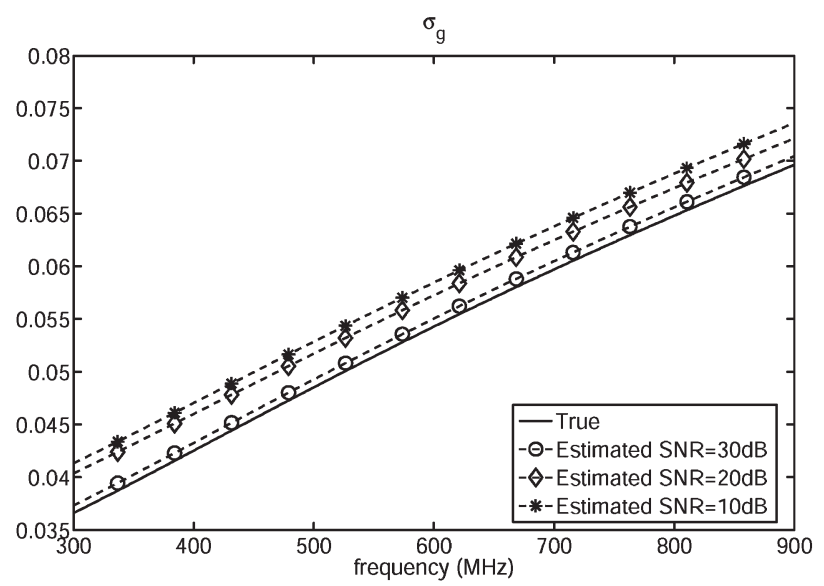

(c)

Fig. 10. True and reconstructed real and imaginary parts of the relative permittivity and real conductivity of the ground for three different SNR ratios versus frequency.

12 iterations, which lasts $210 \mathrm{~s}$ on a $2.8-\mathrm{GHz}$ Pentium IV personal computer using Matlab.

The reconstructed boundaries for 30-dB signal-to-noise ratio (SNR) excitation are also shown in Fig. 6 along with the actual geometry. Increasing the noise level degrades the object reconstruction slightly and has little effect on the airground-interface recovery. Fig. 8 shows the cost-function reduction versus converging simulation steps in logarithmic scale. Fig. 9 demonstrates object reconstruction for all three different SNR ratios.

The estimated ground complex permittivity and real conductivity compared to the true values are presented in Fig. 10 for three various SNR ratios over the frequency range of 300$900 \mathrm{MHz}$. These results show that both real and imaginary parts of permittivity as well as real conductivity are deviated from their actual values as a result of the increase in the noise level.

Table II contains the reconstructed complex permittivity of the object versus SNR ratio. The object complex permittivity is well reconstructed in all three cases.

\section{B. Simulation Study}

We next study the accuracy of the method over a broader range of geometric conditions. Our specific concern is the ability of this approach to localize objects of different sizes
TABLE II

Reconstructed Complex PeRmitTivity of the OBJECt Versus SNR

\begin{tabular}{|c|c|}
\hline SNR $(\mathrm{dB})$ & $\epsilon_{t}$ \\
\hline 30 & $3.0586+i 0.0029$ \\
20 & $3.0693+i 0.0031$ \\
10 & $3.0871+i 0.0035$ \\
\hline
\end{tabular}

and shapes buried under interfaces of varying roughness. As our primary objective here is with the geometric accuracy of the reconstruction, we assume that the dielectric properties are known. For the background, we use Bosnian soil with a density of $1.26 \mathrm{~g} / \mathrm{cc}$ and moisture of $25 \%$ where the complex permittivity of the object is $\epsilon_{t}=2.9(1+i 0.001)$. The incident field is a normal TM-plane wave with frequency in the range of $300-900 \mathrm{MHz}$, separated $100 \mathrm{MHz}$ from each other, and 30-dB SNR ratio. The initial guess for air-ground interface is simply a flat surface, and the object is initiated from a large closed curve residing in the region of interest (illustrated in all example figures).

First, we consider an enlarged almondlike object of first example and assume a rougher ground surface. The object's maximum dimension is about $2 / 3$ of the wavelength at the highest frequency. The geometry is shown in Fig. 11(a). We make use of 21 control points to reconstruct the interface and six control points to reconstruct the large object. Compared 


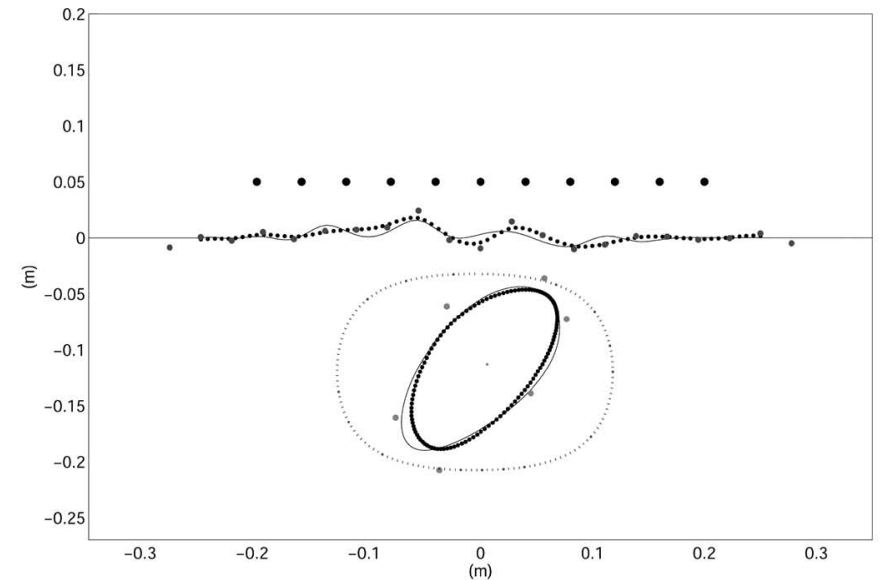

(a)

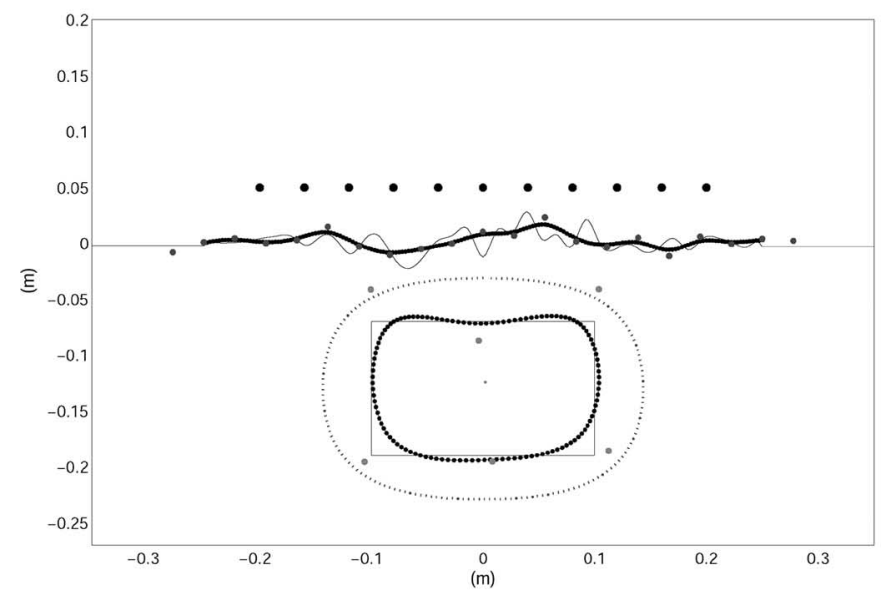

(c)

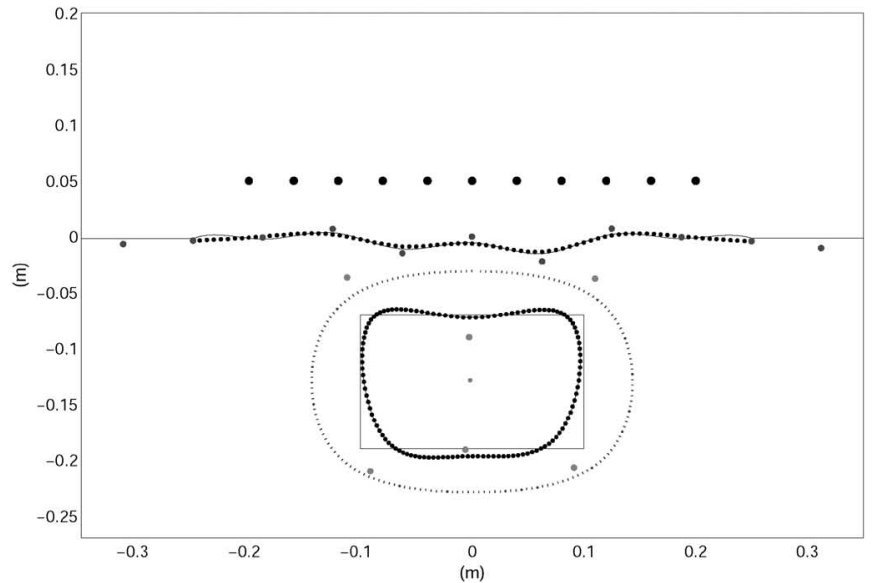

(b)

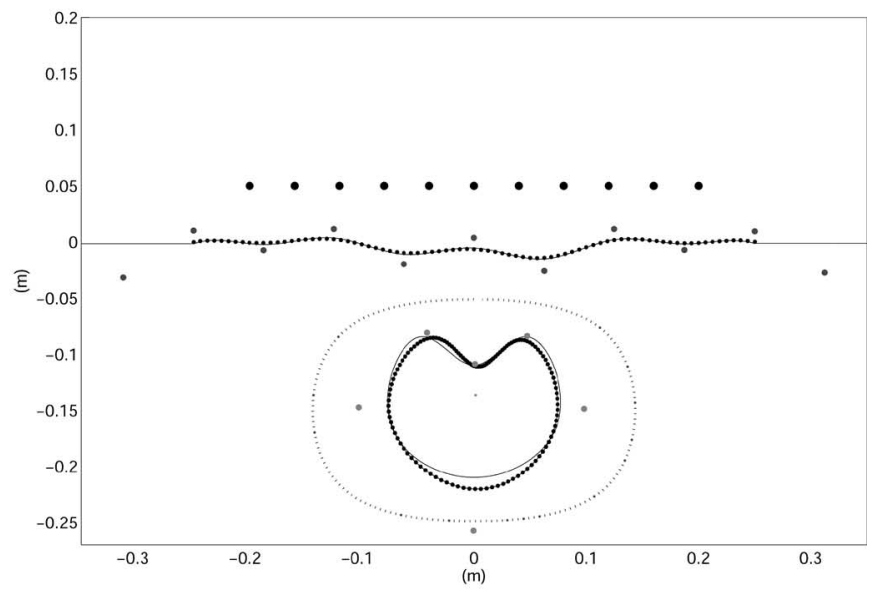

(d)

Fig. 11. Simulation study examples. (a) Large almond-like object with rougher ground surface. (b) Large rectangular object with slightly rough ground surface. (c) Large rectangular object with very rough ground surface. (d) Object with concave edge. In all figures, the true rough surface (solid line) is shown against the estimated rough surface (dotted line) where the control points are represented by large dots. The true object (solid line) is also drawn against the estimated object (dotted line) where the control points are represented by large dots. The larger dotted closed curve is the object initial guess, while the interface initial guess is a straight line at the nominal flat ground surface.

to the results in the previous example, we observe that the geometry of the larger object is recovered about as well. While the reconstruction of the rougher surface does degrade, the loss does not impede our ability to characterize the buried target, which is, after all, the primary objective of the processing.

As a second example, we consider the problem of reconstructing a rectangular box using our smooth splines under surfaces of varying roughness shown in Fig. 11(b) and (c). The object's maximum dimension is about $3 / 4$ of the wavelength at the highest frequency. In both cases, we use normally incident plane waves and six control points to define the object. For the smoother case, we used 11 control points to model the interface while 21 were employed for the rougher ground.

Examining the results in Fig. 11(b) and (c), we can see that the basic size and shape are well captured by our method for both states of surface roughness. As is common with the limited view nature of the problem, the top of the object is recovered more accurately than the bottom. Also, using the fairly small number of control points for the object, we are not able to recover the details of the corners of the box. Still, in the results not shown in this paper, when the surface is smooth and the noise is low, our experiments indicate that we can in fact stably estimate a larger number of control points, and for this box example, we can obtain an accurate representation of the top two corners. Understanding in more detail when such additional detail can be extracted from the data and adapting the parameterization of the object accordingly are an issue we reserve for future effort.

As a final example, in Fig. 11(d), we examine a problem where the object exhibits a concavity. The object's maximum dimension is about half the wavelength at the highest frequency. Using 11 control points for the ground and six for the object, we see that the basic size and location as well as the detail of the concavity are recovered well.

To summarize, in this section, we have examined the proposed inversion method over a broad range of conditions: slightly rough to very rough air-ground interfaces, small and large objects, smooth and sharp object boundaries, and objects with both convex and concave parts. In all cases, the objects are well localized, and their size and shape are characterized with accuracy sufficient for the types of applications where this class of problems arises. Geometrically, the approach clearly works best for objects that are in fact of smooth shape, such as the almond in Figs. 7 and 11(a). For the objects with corners, 
we obtain a more accurate reconstruction of the top than the bottom and can recover the corners with precision only when the interface is flat, and the noise is quite low.

Although not perfect in all situations, we feel that the extensive set of examples presented here provides an honest and thorough understanding of the technique we have developed in this paper and points to a range of interesting issues for future work including: 1) developing an adaptive approach to determining the number of control points required for the object and the interface and 2) improving the SAMM method to better handle a wider range of objects.

\section{CONCLUSION}

A nonlinear optimization method for subsurface sensing of objects buried in the ground with random rough surface was developed. This method reconstructs the boundaries and estimates the dielectric properties of the media simultaneously by comparing the modeled to the true scattered electromagnetic field data. This approach is based on the minimization of the cost function by our nonlinear iterative algorithm using SAMM forward model to compute the scattered fields from the multifrequency and/or multiangle incident plane wave.

In theory, the model-based approach we have pursued can be used to process data arising from any sensor for which we possess a physical model including fly-by ISAR [50], [51], groundcoupled GPR [52]-[54], SAR [55], [56], and medical imaging [57], [58], e.g., 3-D breast-tumor-detection application, which is currently under investigation. The ultimate performance of the method clearly depends on the specifics of the sensor. Optimizing sensor design to maximize the information content in the data is an interesting project, which is beyond the scope of the work in this paper.

In this paper, we used cubic splines to model and reconstruct the boundaries. For future work, we think it would be interesting to examine the use of alternate shape basis functions. Adapting the order of these functions or the number of control points to the information content in the data as well as the frequency of the probing radiation also represents a potentially useful area of exploration. Finally, we note that a locally converging decent-type method was employed in this paper. Although the technique we used for initializing the routine worked well for the applications considered here, we recognize that a last area of future work is the development and validation of global type parameter estimation methods (simulated annealing [59]-[61], genetic methods [62]-[64], particle swarm optimization [65], [66]) either to solve the whole inverse problem, or as a tool for initializing the Levenberg-Marquardt approach.

\section{APPENDIX}

\section{A. Calculating Derivatives of $\mathbf{b}$}

The field difference vector on the boundaries caused by refraction of the incident plane wave is $\mathbf{b}=\left[\mathbf{b}_{1}^{\mathrm{T}}, \mathbf{b}_{2}^{\mathrm{T}}\right]^{\mathrm{T}}$, where the field difference vector at the points on the interface boundary is given by $\mathbf{b}_{1}=\mathbf{b}_{I, 1}+\mathbf{b}_{R, 1}-\mathbf{b}_{T, 1}$, and the transmitted planewave vector on the object boundary in TM case is given by $\mathbf{b}_{2}=\mathbf{b}_{T, 2}$.
The derivative of the vector $\mathbf{b}$ with respect to the unknown vector $\mathbf{u}$ can be written as

$$
\frac{\partial \mathbf{b}}{\partial \mathbf{u}}=\left[\frac{\partial \mathbf{b}}{\partial \mathbf{u}_{1}}, \frac{\partial \mathbf{b}}{\partial \mathbf{u}_{2}}, \frac{\partial \mathbf{b}}{\partial \mathbf{u}_{g}}, \frac{\partial \mathbf{b}}{\partial \mathbf{u}_{t}}\right] .
$$

There is a linear relationship between the coordinates of the points on the boundaries of the curves we are modeling and their associated control points. According to (17), the derivative of each coordinate component of the boundary points with respect to its respective control-point component is equal to its associated B-spline basis function at that point. For a 2-D B-spline, we can write

$$
\begin{aligned}
\frac{\partial x}{\partial p_{x, i}} & =N_{i, k}(x) \\
\frac{\partial y}{\partial p_{y, i}} & =N_{i, k}(y)
\end{aligned}
$$

where $i=1,2, \ldots, N_{u}$.

The vector $\mathbf{b}$ is a function of the boundary-point coordinates in general coordinate system, so the derivatives of elements of vector $\mathbf{b}$ with respect to the interface control-point coordinates $(x, y)$ for $i=1, \ldots, N_{u 1}$ are found using chain rule (A2a)(A2b) and analytical differentiation with respect to the boundary-point coordinates.

The derivatives of $\epsilon_{g}$ with respect to the single-pole model parameters are

$$
\begin{aligned}
\frac{\partial \epsilon_{g}}{\partial \epsilon_{a v}} & =\epsilon_{0} \\
\frac{\partial \epsilon_{g}}{\partial b_{0}} & =z \frac{\partial \epsilon_{g}}{\partial b_{1}}=z^{2} \frac{\partial \epsilon_{g}}{\partial b_{2}}=\frac{i}{\omega\left(1+a_{1} z^{-1}\right)} \\
\frac{\partial \epsilon_{g}}{\partial a_{1}} & =\frac{-i z^{-1}\left(b_{0}+b_{1} z^{-1}+b_{2} z^{-2}\right)}{\omega\left(1+a_{1} z^{-1}\right)^{2}} .
\end{aligned}
$$

The target we use in our experiments is lossy and roughly nondispersive with constant loss tangent, so its frequencyindependent complex permittivity is defined by $\epsilon_{t}=\epsilon_{t r} \epsilon_{0}+$ $i \epsilon_{t i} \epsilon_{0}$. The derivatives of $\epsilon_{t}$ with respect to its real and imaginary parts are

$$
\frac{\partial \epsilon_{t}}{\partial \epsilon_{t r}}=-i \frac{\partial \epsilon_{t}}{\partial \epsilon_{t i}}=\epsilon_{0}
$$

The vector $\mathbf{b}$ is also a function of $\epsilon_{g}$, but not a function of $\epsilon_{t}$. So

$$
\frac{\partial \mathbf{b}}{\partial \mathbf{u}_{t}}=\left[\frac{\partial \mathbf{b}}{\partial \epsilon_{t r}}, \frac{\partial \mathbf{b}}{\partial \epsilon_{t i}}\right]=\mathbf{0}
$$

and

$$
\frac{\partial \mathbf{b}}{\partial \mathbf{u}_{g}}=\frac{\partial \mathbf{b}}{\partial \epsilon_{g}}\left(\frac{\partial \epsilon_{g}}{\partial \mathbf{u}_{g}}\right)^{\mathrm{T}}
$$

where $\partial \epsilon_{g} / \partial \mathbf{u}_{g}$ is found from (A3a)-(A3c), and $\partial \mathbf{b} / \partial \epsilon_{g}$ is calculated as follows. 
The vector $\mathbf{b}_{I}$ is not a function of ground and object permittivities, and the vectors $\mathbf{b}_{R}$ and $\mathbf{b}_{T}$ are just functions of ground permittivity, so

$$
\frac{\partial \mathbf{b}_{I}}{\partial \epsilon_{g}}=\frac{\partial \mathbf{b}_{I}}{\partial \epsilon_{t}}=\frac{\partial \mathbf{b}_{R}}{\partial \epsilon_{t}}=\frac{\partial \mathbf{b}_{T}}{\partial \epsilon_{t}}=0 .
$$

The vectors $\mathbf{b}_{R}$ and $\mathbf{b}_{T}$ are functions of $k_{g y}$, which is a function of $\epsilon_{g}$, so

$$
\begin{aligned}
\frac{\partial \mathbf{b}_{R}}{\partial \epsilon_{g}} & =\frac{\partial \mathbf{b}_{R}}{\partial k_{g y}} \frac{\partial k_{g y}}{\partial \epsilon_{g}}=\mathbf{b}_{R}\left(\frac{-2 k_{a y}}{k_{a y}^{2}-k_{g y}^{2}}\right) \frac{\partial k_{g y}}{\partial \epsilon_{g}} \\
\frac{\partial \mathbf{b}_{T}}{\partial \epsilon_{g}} & =\frac{\partial \mathbf{b}_{T}}{\partial k_{g y}} \frac{\partial k_{g y}}{\partial \epsilon_{g}} \\
\frac{\partial \mathbf{b}_{T}}{\partial k_{g y}} & =\left[-\frac{1}{\mu \omega}\left(+1+k_{g y} \delta\right) \mathbf{E}_{T z}^{\mathrm{T}},-\frac{k_{g x}}{\mu \omega} \delta \mathbf{E}_{T z}^{\mathrm{T}}, \delta \mathbf{E}_{T z}^{\mathrm{T}}\right]^{\mathrm{T}}
\end{aligned}
$$

where

$$
\delta=-\left(\frac{1}{k_{a y}+k_{g y}}+i y\right) .
$$

The derivative $\partial k_{g y} / \partial \epsilon_{g}$ can be obtained analytically.

\section{B. Calculating Derivatives of $\mathbf{L}$}

The derivative of matrix $\mathbf{L}$ with respect to the unknown vector $\mathbf{u}$ can be written as

$$
\frac{\partial \mathbf{L}}{\partial \mathbf{u}}=\left[\frac{\partial \mathbf{L}}{\partial \mathbf{u}_{1}}, \frac{\partial \mathbf{L}}{\partial \mathbf{u}_{2}}, \frac{\partial \mathbf{L}}{\partial \mathbf{u}_{g}}, \frac{\partial \mathbf{L}}{\partial \mathbf{u}_{t}}\right] .
$$

The matrix $\mathbf{L}$ is a function of the coordinates of boundary points in the scattering-center coordinate system: $x^{\prime}=x-x_{0}$ and $y^{\prime}=y-y_{0}$, where $x_{0}$ and $y_{0}$ indicate the object or image scattering-center coordinates and are defined in average form as

$$
x_{0}=\frac{1}{N_{u 2}} \sum_{i=1}^{N_{u 2}} p_{x, i}^{(2)} \quad y_{0}=\frac{1}{N_{u 2}} \sum_{i=1}^{N_{u 2}} p_{y, i}^{(2)} .
$$

The derivatives of elements of $\mathbf{L}$ with respect to the controlpoint components at the object-surface points are found using chain rule (A2a), (A2b) and analytical differentiation with respect to boundary-point coordinates using Bessel functions recurring derivative relations

$$
\begin{aligned}
& \frac{\partial G_{n}\left(x^{\prime}, y^{\prime}\right)}{\partial x^{\prime}}=\left(k \cos \phi G_{n-1}\left(x^{\prime}, y^{\prime}\right)-\frac{n}{\rho} G_{n}\left(x^{\prime}, y^{\prime}\right)\right) e^{i \phi} \\
& \frac{\partial G_{n}\left(x^{\prime}, y^{\prime}\right)}{\partial y^{\prime}}=\left(k \sin \phi G_{n-1}\left(x^{\prime}, y^{\prime}\right)+\frac{i n}{\rho} G_{n}\left(x^{\prime}, y^{\prime}\right)\right) e^{i \phi}
\end{aligned}
$$

with $\rho=\sqrt{x^{\prime 2}+y^{\prime 2}}$, and $\phi=\tan ^{-1}\left(y^{\prime} / x^{\prime}\right)$.

The matrix $\mathbf{L}$ is a function of $\epsilon_{g}$ and $\epsilon_{t}$, such that

$$
\frac{\partial \mathbf{L}}{\partial \mathbf{u}_{g}}=\frac{\partial \mathbf{L}}{\partial \epsilon_{g}}\left(\frac{\partial \epsilon_{g}}{\partial \mathbf{u}_{g}}\right)^{\mathrm{T}}
$$

where $\partial \epsilon_{g} / \partial \mathbf{u}$ is found from (A3a)-(A3c).
To obtain the derivatives of $\mathbf{L}$ with respect to the complex permittivity, we differentiate (13a)-(13c) and take advantage of the following Bessel-function derivative

$$
\frac{\partial G_{n}\left(x^{\prime}, y^{\prime}\right)}{\partial \epsilon_{g}}=\left(\rho G_{n-1}\left(x^{\prime}, y^{\prime}\right)-\frac{n}{k_{g}} G_{n}\left(x^{\prime}, y^{\prime}\right)\right) \frac{k_{g}}{2 \epsilon_{g}}
$$

where $k_{g}=2 \pi f \sqrt{\mu \epsilon_{g}}$.

Similar equations are satisfied for derivative with respect to $\epsilon_{t}$, and we obtain

$$
\frac{\partial \mathbf{L}}{\partial \mathbf{u}_{t}}=\left[\frac{\partial \mathbf{L}}{\partial \epsilon_{t r}}, \frac{\partial \mathbf{L}}{\partial \epsilon_{t i}}\right]=\left[\frac{\partial \mathbf{L}}{\partial \epsilon_{t}} \epsilon_{0}, i \frac{\partial \mathbf{L}}{\partial \epsilon_{t}} \epsilon_{0}\right] .
$$

\section{Calculating Derivatives of $\mathbf{M}$}

The matrix $\mathbf{M}$ is a function of the distance between receivers' locations and object scattering center: $x^{\prime}=x-x_{0}$ and $y^{\prime}=y-y_{0}$, where $x$ and $y$ represent receiver-location components. Applying the chain rule, using (A2a), (A2b) and taking derivatives of elements of $\mathbf{M}$ with respect to the controlpoint components at the receivers, $\partial \mathbf{M} / \partial \mathbf{u}_{1}$ and $\partial \mathbf{M} / \partial \mathbf{u}_{2}$ are obtained. The elements of matrices $\partial \mathbf{M} / \partial x^{\prime}$ and $\partial \mathbf{M} / \partial y^{\prime}$ are similar to $\partial \mathbf{L} / \partial x^{\prime}$ and $\partial \mathbf{L} / \partial y^{\prime}$, but here, $x^{\prime}$ and $y^{\prime}$ indicate distance between the receivers and the object scattering center. The derivatives $\partial \mathbf{M} / \partial \mathbf{u}_{g}$ and $\partial \mathbf{M} / \partial \mathbf{u}_{t}$ satisfy equations similar to (A15) and (A17) at the receivers.

\section{REFERENCES}

[1] L. Peters, Jr., J. J. Daniels, and J. D. Young, "Ground penetrating radar as a subsurface environmental sensing tool," Proc. IEEE, vol. 82, no. 12, pp. 1802-1822, Dec. 1994.

[2] J. Zhang, R. L. Mackie, and T. R. Madden, "3-D resistivity forward modeling and inversion using conjugate gradients," Geophysics, vol. 60, no. 5, pp. 1313-1325, Sep./Oct. 1995.

[3] D. J. LaBrecque, M. Miletto, W. Daily, A. Ramirez, and E. Owen, "The effects of noise on Occam's inversion of resistivity tomography data," Geophysics, vol. 61, no. 2, pp. 538-548, 1996.

[4] T. T. N. Lan, U. Seidel, and H. G. Walther, "Theory of microstructure depth profiling by photothermal measurement," J. Appl. Phys., vol. 77, no. 9, pp. 4739-4745, May 1, 1995.

[5] A. Mandelis, F. Funak, and M. Munidasa, "Generalized methodology for thermal diffusivity depth profile reconstruction in semi-infinite and finitely thick inhomogeneous solids," J. Appl. Phys., vol. 80, no. 10, pp. 5570-5578, Nov. 15, 1996.

[6] L. Nicolaides and A. Mandelis, "Image-enhanced thermal-wave slice diffraction tomography with numerically simulated reconstructions," Inverse Probl., vol. 13, no. 5, pp. 1339-1412, 1997.

[7] W. C. Chew and Y. M. Wang, "Reconstruction of two-dimensional permittivity distribution using the distorted Born iterative method," IEEE Trans. Med. Imag., vol. 9, no. 2, pp. 218-225, Jun. 1990.

[8] M. E. Kilmer, E. L. Miller, D. A. Boas, and D. Brooks, "A shape-based reconstruction technique for DPDW data, Invited paper," Opt. Express, vol. 7 , no. 13 , pp. 481-491, Dec. 2000.

[9] E. L. Miller, M. Kilmer, and C. M. Rappaport, "A new shape-based method for object localization and characterization from scattered field data," IEEE Trans. Geosci. Remote Sens., vol. 38, no. 4, pp. 1682-1696, Jul. 2000.

[10] T. Dogaru and L. Carin, "Time-domain sensing of targets buried under a rough air-ground interface," IEEE Trans. Antennas Propag., vol. 46, no. 3, pp. 360-372, Mar. 1998.

[11] H. Zhan, C. M. Rappaport, M. El-Shenawee, and E. L. Miller, "Mine detection under rough ground surfaces using 2-D FDTD modeling and hypothesis testing," in Proc. IEEE Int. Symp. Antennas Propag., Boston, MA, Jul. 8-13, 2001, vol. 3, p. 756. 
[12] O. Dorn, E. L. Miller, and C. M. Rappaport, "A shape reconstruction method for electromagnetic tomography using adjoint fields and level sets," Inverse Probl., vol. 16, no. 5, pp. 1119-1156, Oct. 2000.

[13] Y. M. Wang and W. C. Chew, "An iterative solution of the twodimensional electromagnetic inverse scattering problem," Int. J. Imaging Syst. Technol., vol. 1, pp. 100-108, 1989.

[14] V. Galdi, H. Feng, D. Castañon, W. C. Karl, and L. B. Felsen, "Moderately rough surface underground imaging via short-pulse quasi-ray Gaussian beams," IEEE Trans. Antennas Propag., vol. 51, no. 9, pp. 2304-2318, Sep. 2003.

[15] H. Feng, W. C. Karl, and D. A. Castañon, "A curve evolution approach to object-based tomographic reconstruction," IEEE Trans. Image Process., vol. 12, no. 1, pp. 44-57, Jan. 2003.

[16] F. Santosa, "A level-set approach for inverse problems involving obstacles," ESAIM: Control Optim. Calc. Var., vol. 1, pp. 17-33, 1996.

[17] R. A. Weisenseel, W. C. Karl, D. A. Castañon, E. L. Miller, C. M. Rappaport, and C. A. DiMarzio, "Statistical fusion of GPR and EMI data," in Proc. Detection and Remediat. Technol. Mines and Minelike Targets IV, A. C. Dubey, J. F. Harvey, J. Broach, and R. E. Dugan, Eds., Aug. 1999, vol. 3710, pp. 1179-1187.

[18] X. Xu, E. L. Miller, C. M. Rappaport, and G. Sower, "Statistical method to detect subsurface objects using array ground penetrating radar data," IEEE Trans. Geosci. Remote Sens., vol. 40, no. 4, pp. 963-976, Apr. 2002.

[19] T. Dogaru, L. Collins, and L. Carin, "Optimal time-domain detection of a deterministic target buried under a randomly rough interface," IEEE Trans. Antennas Propag., vol. 49, no. 3, pp. 313-326, Mar. 2001.

[20] M. Belge, M. Kilmer, and E. L. Miller, "Wavelet domain image restoration with adaptive edge-preserving regularization," IEEE Trans. Image Process., vol. 9, no. 4, pp. 597-608, Apr. 2000.

[21] M. Cetin and W. C. Karl, "Feature-enhanced synthetic aperture radar image formation based on non-quadratic regularization," IEEE Trans. Image Process., vol. 10, no. 4, pp. 623-631, Apr. 2001.

[22] C. R. Vogel and M. E. Oman, "Fast, robust total variation-based reconstruction of noisy, blurred images," IEEE Trans. Image Process., vol. 7, no. 7, pp. 813-824, Jul. 1998.

[23] A. Sahin and E. L. Miller, "Model-based multiple object detection using high-resolution near field array processing," IEEE Trans. Geosci. Remote Sens., vol. 39, no. 1, pp. 136-141, Jan. 2001.

[24] A. Litman, D. Lesselier, and F. Santosa, "Reconstruction of a two-dimensional binary obstacle by controlled evolution of a level-set," Inverse Probl., vol. 14, no. 3, pp. 685-706, Jun. 1998.

[25] A. W. Morgenthaler and C. M. Rappaport, "Semianalytic mode matching for detecting nonmetallic mines buried in realistic soils," in Proc. SPIE Aerosense Conf., Orlando, FL, 2000, pp. 1149-1159.

[26] — , "Scattering from dielectric objects buried beneath randomly rough ground: Validating the semianalytic mode matching algorithm with 2-D FDFD," IEEE Trans. Geosci. Remote Sens., vol. 39, no. 11, pp. 2421-2428, Nov. 2001.

[27] M. Sadiko, Numerical Techniques in Electromagnetics. Boca Raton, FL: CRC Press, 1992, pp. 135-204.

[28] R. Harrington, Field Computation by Moment Method. New York: MacMillan, 1968.

[29] S. Vitebskiy and L. Carin, "Moment-method modeling of shortpulse scattering from and the resonances of a wire buried inside a lossy, dispersive half-space," IEEE Trans. Antennas Propag., vol. 43, no. 11, pp. 1303-1312, Nov. 1995.

[30] C. Rappaport, S. Wu, and S. Winton, "FDTD wave propagation in dispersive soil using a single pole conductivity model," IEEE Trans. Magn., vol. 35, no. 3, pp. 1542-1545, May 1999.

[31] K. Arbter, W. E. Snyder, H. Burkhardt, and G. Hirzinger, "Application of affine-invariant Fourier descriptors to recognition of 3-D objects," IEEE Trans. Pattern Anal. Mach. Intell., vol. 12, no. 7, pp. 640-647, Jul. 1990.

[32] E. T. Whittaker and G. Robinson, "Lagrange's formula of interpolation," in 17 in The Calculus of Observations: A Treatise on Numerical Mathematics, 4th ed. New York: Dover, 1967, pp. 28-30.

[33] W. C. Karl, G. C. Verghese, and A. S. Willsky, "Reconstructing ellipsoids from projections," Comput. Vis., Graph., Image Process.: Graph. Models Image Process., vol. 56, no. 2, pp. 124-139, Mar. 1994.

[34] M. E. Kilmer, E. L. Miller, A. Barbaro, and D. Boas, "3D shape-based imaging for diffuse optical tomography," Appl. Opt., vol. 42, pp. 3129$3144,2003$.

[35] C. Boor, "On calculation with B-splines," J. Approx. Theory, vol. 6, no. 1, pp. 50-62, 1972.

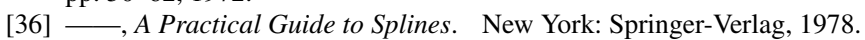

[37] L. L. Shumaker, Spline Functions: Basic Theory. New York: Wiley, 1981.
[38] E. L. Miller and A. S. Willsky, "Multiscale, statistical anomaly detection analysis and algorithms for linearized inverse scattering problems," Multidimens. Syst. Signal Process., vol. 8, no. 1/2, pp. 151-184, Jan. 1997.

[39] _ - "Wavelet-based methods for the nonlinear inverse scattering problem using the extended born approximation," Radio Sci., vol. 31, no. 1, pp. 51-67, 1996.

[40] ㄴ , "A multiscale, statistically-based inversion scheme for the linearized, inverse scattering problem," IEEE Trans. Geosci. Remote Sens., vol. 34, no. 2, pp. 346-357, Mar. 1996.

[41] D. H. Staelin, A. W. Morgenthaler, and J. A. Kong, Electromagnetic Waves. Englewood Cliffs, NJ: Prentice-Hall, 1994, pp. 157-160.

[42] J. A. Kong, Electromagnetic Wave Theory. New York: Wiley, 1986, pp. 485-489.

[43] J. A. Stratton, Electromagnetic Theory. New York: McGraw-Hill, 1941, pp. 392-419.

[44] K. Levenberg, "A method for the solution of certain nonlinear problems in least squares," Quart. Appl. Math., vol. 2, no. 7, pp. 164-168, 1944.

[45] D. Marquardt, "An algorithm for least-squares estimation of nonlinear parameters," SIAM J. Appl. Math., vol. 11, no. 2, pp. 431-441, 1963.

[46] C. T. Kelley, Iterative Methods for Optimization. Philadelphia, PA: SIAM, 1999.

[47] J. Nocedal and S. J. Wright, Numerical Optimization. New York: Springer-Verlag, 1999.

[48] C. Rappaport, M. Kilmer, and E. Miller, "Accuracy considerations in using the PML ABC with FDFD Helmholtz equation computation," Int. J. Numer. Model., vol. 13, no. 471, pp. 471-482, Sep. 2000.

[49] C. Rappaport. (2001, Mar.). FDTD Wave Propagation in Dispersive Soil Using a Single Pole Conductivity Model, Northeastern University, Boston, MA. [Online]. Available: http://www.censsis.neu.edu/muri/ soilmodel/soilindex.html

[50] G. Dural and D. L. Moffatt, "ISAR imaging to identify basic scattering mechanisms," IEEE Trans. Antennas Propag., vol. 42, no. 1, pp. 99-110, Jan. 1994.

[51] G. Wang, X.-G. Xia, and V. C. Chen, "Three-dimensional ISAR imaging of maneuvering targets using three receivers," IEEE Trans. Image Process., vol. 10, no. 3, pp. 436-447, Mar. 2001.

[52] T. Saarenketo and P. Roimela, "Ground penetrating radar technique in asphalt pavement density quality control," in Proc. 7th Int. Conf. Ground Penetrating Radar, Lawrence, KS, May 27-30, 1998, pp. 461-466.

[53] F. Roth, P. van Genderen, and M. Verhaegen, "Convolutional models for buried target characterization with ground penetrating radar," IEEE Trans. Antennas Propag., vol. 53, no. 11, pp. 3799-3810, Nov. 2005.

[54] P. Gamba and S. Lossani, "Neural detection of pipe signatures in ground penetrating radar images," IEEE Trans. Geosci. Remote Sens., vol. 38, no. 2, pp. 790-797, Mar. 2000.

[55] L. Carin, R. Kapoor, and C. E. Baum, "Polarimetric SAR imaging of buried landmines," IEEE Trans. Geosci. Remote Sens., vol. 36, no. 6, pp. 1985-1988, Nov. 1998.

[56] R. B. Cosgrove, P. Milanfar, and J. Kositsky, "Trained detection of buried mines in SAR images via the deflection-optimal criterion," IEEE Trans. Geosci. Remote Sens., vol. 42, no. 11, pp. 2569-2575, Nov. 2004.

[57] P. M. Meaney, M. W. Fanning, D. Li, S. P. Poplack, and K. D. Paulsen, "A clinical prototype for active microwave imaging of the breast," IEEE Trans. Microw. Theory Tech., vol. 48, no. 11, pp. 1841-1853, Nov. 2000.

[58] B. Zhou, W. Shao, and G. Wang, "On the resolution of UWB microwave imaging of tumors in random breast tissue," in Proc. IEEE Int. Symp. Antennas and Propag. Soc., Jul. 3-8, 2005, vol. 3A, pp. 831-834.

[59] A. K. Mandal, S. Pal, A. K. De, and S. Mitra, "Novel approach to identify good tracer clouds from a sequence of satellite images," IEEE Trans. Geosci. Remote Sens., vol. 43, no. 4, pp. 813-818, Apr. 2005.

[60] S. Gudmundsson, J. M. Carstensen, and F. Sigmundsson, "Unwrapping ground displacement signals in satellite radar interferograms with aid of GPS data and MRF regularization," IEEE Trans. Geosci. Remote Sens., vol. 40, no. 8, pp. 1743-1754, Aug. 2002.

[61] J. Schou and H. Skriver, "Restoration of polarimetric SAR images using simulated annealing," IEEE Trans. Geosci. Remote Sens., vol. 39, no. 9, pp. 2005-2016, Sep. 2001.

[62] W. H. Slade, H. W. Ressom, M. T. Musavi, and R. L. Miller, "Inversion of ocean color observations using particle swarm optimization," IEEE Trans. Geosci. Remote Sens., vol. 42, no. 9, pp. 1915-1923, Sep. 2004.

[63] B.-K. Jeon, J.-H. Jang, and K.-S. Hong, "Road detection in spaceborne SAR images using a genetic algorithm," IEEE Trans. Geosci. Remote Sens., vol. 40, no. 1, pp. 22-29, Jan. 2002.

[64] A. Qing, C. K. Lee, and L. Jen, "Electromagnetic inverse scattering of two-dimensional perfectly conducting objects by real-coded genetic algorithm," IEEE Trans. Geosci. Remote Sens., vol. 39, no. 3, pp. 665-676, Mar. 2001. 
[65] M. Donelli, G. Franceschini, A. Martini, and A. Massa, "An integrated multiscaling strategy based on a particle swarm algorithm for inverse scattering problems," IEEE Trans. Geosci. Remote Sens., vol. 44, no. 2, pp. 298-312, Feb. 2006

[66] B. C. K. Tso and P. M. Mather, "Classification of multisource remote sensing imagery using a genetic algorithm and Markov random fields," IEEE Trans. Geosci. Remote Sens., vol. 37, no. 3, pp. 1255-1260, May 1999.

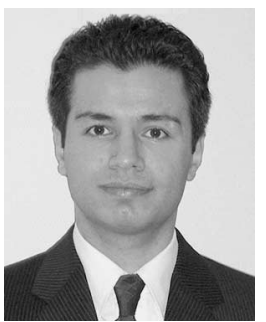

Reza Firoozabadi ( $\left.\mathrm{S}^{\prime} 02\right)$ received the B.S. degree from the Sharif University of Technology, Tehran, Iran, in 1996 and the M.S. degree from the Amirkabir University of Technology, Tehran, in 1999, both in electrical engineering. He is currently working toward the Ph.D. degree in electrical engineering at Northeastern University, Boston, MA

He worked as a Research Assistant for several years in the Microwave/Millimeter-wave and Wireless Communication Laboratory in the Electrical Engineering Department, Amirkabir University of Technology. Since March 2002, he has been a Research Assistant with the Department of Electrical Engineering and the Center for Subsurface Sensing and Imaging Systems, Northeastern University. His research interests include inverse problems and signal-processing techniques, computational electromagnetics, high-frequency electronics, microwave/MM-wave circuit and system design, and electromagnetic modeling and analysis of active devices and circuits.

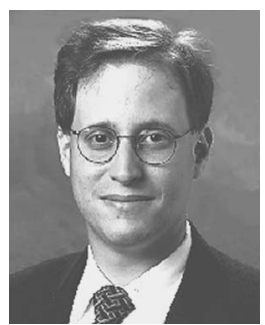

Eric L. Miller (S'90-M'95-SM'03) received the S.B., S.M., and Ph.D. degrees from the Massachusetts Institute of Technology, Cambridge, in 1990, 1992, and 1994, respectively, all in electrical engineering and computer science.

$\mathrm{He}$ is currently a Professor with the Department of Electrical and Computer Engineering, Northeastern University, Boston, MA. His research interests include physics-based tomographic image formation and object characterization, inverse problems in general and inverse scattering in particular, regularization, statistical signal and imaging processing, and computational physical modeling. This work has been carried out in the context of applications including medical imaging, nondestructive evaluation, environmental monitoring and remediation, landmine and unexploded ordnance remediation, and automatic target detection and classification.

Dr. Miller received the CAREER Award from the National Science Foundation in 1996 and the Outstanding Research Award from the College of Engineering at Northeastern University in 2002. From 1998 to 2002, he served as an Associate Editor for the IEEE TRANS ACTIONS ON IMAGE PROCESSING. $\mathrm{He}$ is currently serving as an Associate Editor for the IEEE TRANSACTIONS on Geoscience and Remote Sensing. He is the Cogeneral Chair of the 2008 IEEE International Geoscience and Remote Sensing Symposium to be held in Boston, MA. He is a member of Tau Beta Pi, Phi Beta Kappa, and Eta Kappa Nu.

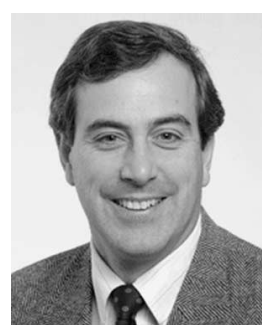

Carey M. Rappaport (M'87-SM'96-F'06) received the S.B. degree in mathematics, the S.B. S.M., and E.E. degrees in electrical engineering, in 1982, and the Ph.D. degree in electrical engineering, in 1987, from the Massachusetts Institute of Technology (MIT), Cambridge.

He has worked as a Teaching and Research Assistant with MIT from 1981 to 1987 and during the summers at COMSAT Laboratories in Clarksburg, $\mathrm{MD}$, and The Aerospace Corporation in El Segundo, CA. He joined the faculty at Northeastern University in Boston, MA, in 1987. He has been a Professor of electrical and computer engineering since July 2000. During the fall of 1995, he was a Visiting Professor of electrical engineering at the Electromagnetics Institute of the Technical University of Denmark, Lyngby, as part of the W. Fulbright International Scholar Program. During the second half of 2005, he was a Visiting Research Scientist at the Commonwealth Scientific Industrial and Research Organization, Epping, Australia. He has consulted for Geo-Centers, Inc., PPG, Inc., and several municipalities on wave propagation and modeling, and microwave heating and safety. He was Principal Investigator of an Army Research Office (ARO)-sponsored Multidisciplinary University Research Initiative on Humanitarian Demining and Co-Principal Investigator of the NSF-sponsored Center for Subsurface Sensing and Imaging Systems (CenSSIS) Engineering Research Center. He has authored over 200 technical journal and conference papers in the areas of microwave antenna design, electromagnetic wave propagation and scattering computation, and bioelectromagnetics. He has received two reflector antenna patents, two biomedical device patents, and three subsurface-sensing device patents.

Dr. Rappaport was awarded the IEEE Antenna and Propagation Society's H.A. Wheeler Award for best applications paper, as a student in 1986. He is a member of the Sigma Xi and Eta Kappa Nu professional honorary societies.

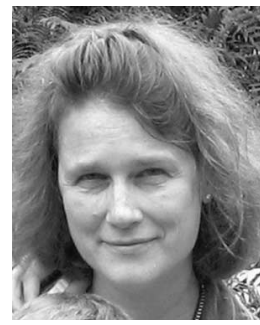

Ann W. Morgenthaler received the B.A. in physics from Harvard University, Cambridge, MA, and the E.E. degree in electrical engineering and computer science from Massachusetts Institute of Technology (MIT), Cambridge.

She is an author of the textbook "Electromagnetic Waves" with David Staelin and Jin Au Kong. Her research interests include fast forward modeling techniques for ground-penetrating radar scattering from realistic objects in lossy media under rough ground surfaces. 